ARTICLE LINK: http://www.sciencedirect.com/science/article/pii/S019126151500048X

PLEASE CITE THIS ARTICLE AS

Han, K., Gayah, V.V., 2015. Continuum signalized junction model for dynamic traffic networks: Offset, spillback, and multiple signal phases. Transportation Research Part B 77, 213-239.

\title{
Continuum signalized junction model for dynamic traffic networks: Offset, spillback, and multiple signal phases
}

\author{
Ke Han ${ }^{\mathrm{a}, *}$, Vikash V. Gayah ${ }^{\mathrm{b}}$ \\ ${ }^{a}$ Department of Civil and Environmental Engineering, Imperial College London, United Kingdom. \\ ${ }^{b}$ Department of Civil and Environmental Engineering, Pennsylvania State University, USA.
}

\begin{abstract}
This paper extends the continuum signalized intersection model exhaustively studied in Han et al. (2014) to more accurately account for three realistic complications: signal offsets, queue spillbacks, and complex signal phasing schemes. The model extensions are derived theoretically based on signal cycle, green split, and offset, and are shown to approximate well traffic operations at signalized intersections treated using the traditional (and more realistic) on-and-off model. We propose a generalized continuum signal model, which explicitly handles complex vehicle spillback patterns on signalized networks with provable error estimates. Under mild conditions, the errors are small and bounded by fixed values that do not grow with time. Overall, this represents a significant improvement over the original continuum model, which had errors that grew quickly with time in the presence of any queue spillbacks and for which errors were not explicitly derived for different offset cases. Thus, the new model is able to more accurately approximate traffic dynamics in large networks with multiple signals under more realistic conditions. We also qualitatively describe how this new model can be applied to several realistic intersection configurations that might be encountered in typical urban networks. These include intersections with multiple entry and exit links, complex signal phasing, all-red times, and the presence of dedicated turning lanes. Numerical tests of the models show remarkable consistency with the on-and-off model, as expected from the theory, with the added benefit of significant computational savings and higher signal control resolution when using the continuum model.
\end{abstract}

Keywords: traffic signal, continuum approximation, LWR model, offset, spillback, multiple stages

\section{Introduction}

Signalized intersections are typically the most restrictive bottlenecks in urban environments. For this reason, the performance of individual arterials or small networks is usually described by the operations at the signalized intersections that make up these facilities (TRB, 2000). These locations also tend to be the primary focus of local and network-wide urban traffic control strategies (Miller, 1963; Robertson and Bretherton, 1974; Shelby, 2004; Chitour and Piccoli, 2005; Gayah and Daganzo, 2012; Guler and Cassidy, 2012; Gu et al., 2014). Therefore, accurate models of traffic dynamics at individual signalized intersections are essential to the design, management and control of large urban transportation networks.

\footnotetext{
${ }^{*}$ Corresponding author

Email addresses: k.han@imperial.ac.uk (Ke Han ), gayah@engr.psu.edu (Vikash V. Gayah)
} 


\section{ARTICLE LINK: http://www.sciencedirect.com/science/article/pii/S019126151500048X}

\section{PLEASE CITE THIS ARTICLE AS}

Han, K., Gayah, V.V., 2015. Continuum signalized junction model for dynamic traffic networks: Offset, spillback, and multiple signal phases. Transportation Research Part B 77, 213-239.

Unfortunately, operations at signalized intersections are complicated by the cyclical on-and-off pattern of traffic signals. While the pattern is conceptually simple - vehicles are allowed to move through the signal during the green phase or 'on' period and are restricted from moving during the red phase or 'off' period - the periodic switching between these two distinct phases must be tracked to accurately model vehicle throughput. Binary variables are typically used to track the phase of the traffic signal assigned to each approach of every signalized intersection. However, incorporating many binary variables into the optimization frameworks used to study large-scale urban networks (such as Improta and Cantarella (1984) and Lo (1999a,b)) results in complex mixed integer mathematical programs (MIMPs) that are difficult to solve exactly. Instead, heuristic optimization methods must be used (Foy et al., 1992; Chiu and Chand, 1993; Ceylan and Bell, 2004; Murat and Gedizlioglu, 2005), but these provide inexact and suboptimal solutions. Even when exact methods are available to solve MIMPs, they are typically time intensive and computational expensive. The discrete on-and-off nature of traffic signals also results in discontinuous travel time functions with discrete jumps that make dynamic traffic assignment models (Friesz et al., 1993, 2013) difficult to implement. This means that the combined treatment of dynamic traffic assignment with signal control (DUESC) becomes especially difficult to solve (Aziz and Ukkusuri, 2012; Ukkusuri et al, 2013).

To overcome these challenges, recent studies (Smith, 2010; Ge and Zhou, 2012) have proposed a continuum model to approximate traffic dynamics at a signalized intersection without the need for distinct signal phases. A fraction, $\eta$, of the downstream link's capacity is assumed to be available to vehicles discharging from a given approach during the entire signal cycle. In this simple model, $\eta$, is assumed to be equal to the proportion of the cycle allocated to the subject approach for movement through the intersection. This continuum model has a number of advantages over the on-and-off approach: (1) it requires fewer integer variables when modeling dynamics on large-scale networks, which decreases the computational complexity of modeling and optimization processes; (2) it provides more flexibility in selecting the time resolution in a discrete-time environment; (3) it eliminates discontinuities in travel time functions, which allows dynamic user equilibrium problems (Friesz et al., 2013) to be formulated in more exact ways without the need to introduce indifference behaviors (Szeto and Lo, 2006; Ge and Zhou, 2012; Han, 2013); and (4) it imposes fewer implicit constraints on potential signal timings in optimization procedures. More details on these advantages are provided in Han et al. (2014). However, the primary drawback of this approach is that it does not exactly replicate the dynamics that would arise at an intersection. If significant errors exist between the two approaches, the continuum representation becomes invalid. This model appears to be an extension of the 'store-and-forward' model (SFM), originally proposed by Gazis and Potts (1963), which assumes flows discharge continuously through an intersection movement at a rate equal to the product of the green ratio and saturation flow. The SFM is restrictive in that it assumes that the signal is always completely saturated. Aboudolas et al. (2009) extended the SFM to consider under-saturated conditions and complete flow blockages from downstream queues. However, this model is still deficient as it fails to account for partial disruptions that might occur due to spillback with queued flows greater than zero. Furthermore, it cannot accommodate cycle lengths and offsets as variables in signal timing optimization procedures.

Recently, Han et al. (2014) performed a comprehensive comparison between the continuum model (Smith, 2010; Ge and Zhou, 2012) and the on-and-off signal model. By applying the Hamilton-Jacobi formulation and generalized Lax-Hopf formula (Aubin et al., 2008; Claudel and Bayen, 2010) to the Lighthill-Whitham-Richards model of traffic on links (Lighthill and Whitham, 1955; Richards, 1956), the authors were able to quantify the maximum errors that arise within the continuum model when compared with the exact on-and-off model. This study found that the continuum model works quite well in the absence of queue spillback to the subject intersection. In this case, errors in predicted vehicle counts between this approximation and the exact on-off model are bounded and quite small. However, the approximation does not perform well when queue spillback occurs. For sustained spillback that lasts multiple cycles, errors are large and grow with time. The magnitude of these errors are generally smaller when using a strictly concave fundamental diagram as opposed to a triangular or trapezoidal fundamental diagram, although those errors still grow with time and are unbounded. Moreover, the continuum model does not accurately approximate cases with transient spillbacks that occur entirely during the duration of a signal's cycle. Both sustained and transient spillback frequently arises in congested urban networks, ${ }^{1}$ and these limitations reduce the applicability of the continuum signal model. The continuum model studied in that paper also fails to account for offsets between adjacent signals. These offsets significantly impact vehicle travel times along a long arterial that contains many signalized intersections. Furthermore, the continuum model was only proposed for fairly simplistic intersection configurations.

\footnotetext{
${ }^{1} \mathrm{~A}$ concrete example of the sustained and transient spillbacks is presented in Section 4.1 of this paper.
} 


\section{ARTICLE LINK: http://www.sciencedirect.com/science/article/pii/S019126151500048X}

\section{PLEASE CITE THIS ARTICLE AS}

Han, K., Gayah, V.V., 2015. Continuum signalized junction model for dynamic traffic networks: Offset, spillback, and multiple signal phases. Transportation Research Part B 77, 213-239.

In light of this, the purpose of this study is to extend the simple continuum signal model studied in Han et al. (2014) to account for these more realistic complications. Specifically, a modification of the original continuum model is theoretically derived to accommodate signal timing offsets between adjacent signals along a corridor, assuming the absence of vehicle spillback. Moreover, a generalized continuum signal model (GCSM) is proposed to handle the presence of both sustained and transient queue spillbacks in the most general setting, which also takes into account signal offsets. Notably, the GCSM subsumes the Han et al. (2014) model as a special case and is theoretically proven to accurately approximate complex dynamics on signalized networks regardless of the presence of spillbacks. It can even handle spillbacks that affect multiple junctions at the same time, which is not considered in any existing continuum signal model. Qualitative discussions are also provided to demonstrate how the model can be applied to realistic intersection configurations that have multiple entry or exit links, complex signal phasing schemes, all-red times and dedicated turn-lanes. Using these extensions, the new continuum model can be used to study more realistic networks with increased accuracy, while maintaining many of the benefits of the approximate approaches outlined above. These benefits include: reduced computational complexity in modeling and optimization, more flexibility in selection of time resolutions, and the elimination of discontinuities in travel time functions for DTA applications. The derivation of these extensions rely on the assumption of a triangular or trapezoidal fundamental diagram that relates flow to density at points on a link. However, such functional forms of the fundamental diagram have been shown to be realistic in analysis of empirical traffic data (Cassidy, 1998) and has been used extensively for modeling purposes (Daganzo, 1994, 1995; Newell, 1993), even in urban areas near signalized intersections (Gu et al., 2014).

This work presents a significant improvement of the previously studied continuum models in terms of approximating the on-and-off representation of network-wide signal controls. Specifically, our model is the first to explicitly treat both the no-spillback and sustained/transient spillback cases with the knowledge of offsets between adjacent signals along a corridor and cycle lengths, and substantially reduces the errors that arise in these cases as derived in theory and validated through numerical tests. In particular, the error for the sustained/transient spillback case is uniformly bounded and does not grow with time under minor assumptions, unlike the Han et al. (2014) model. Extensive numerical simulations on a test network show that errors in predicted cumulative vehicle count curves can be reduced by up to $82 \%$ when compared to the original model presented in Han et al. (2014). Overall, the findings suggest that the proposed continuum approach is a promising method to simplify modeling representations of large-scale urban traffic networks. It should allow these complex systems to be studied in a relatively simple way with much less computational effort while still maintaining the temporal and spatial properties of congestion dynamics in networks.

The specific contributions of this paper are summarized as follows:

1. A method is derived to incorporate offsets between adjacent traffic signals in the continuum signal model in the absence of spillbacks. This method requires that the phasing scheme and offsets are known a priori, which are common assumptions for most transportation applications.

2. The generalized continuum signal model (GCSM) is derived to incorporate the impacts of sustained and transient spillbacks on networks of arbitrary size and configuration. The GCSM subsumes the no-spillback model (Han et al., 2014) as a special case. This model relies on the knowledge of the phasing scheme, signal offsets, and traffic properties on the links.

3. Error bounds are theoretically derived for all the previous methods, which suggest a substantial reduction in the approximation errors, compared to the Han et al. (2014) model. The signal offset and queue spillback models are rigorously tested using a battery of simulations. The simulation results show that the proposed continuum signal models approximate the on-and-off signal model remarkably well for a range of conditions.

4. Instructions are provided to implement complex phasing schemes at a realistic signalized intersection within the continuum signal model.

5. The benefits of using the continuum model instead of the on-off one are demonstrated quantitatively with several simulation and optimization problems. The continuum model's improved computational efficiency in straightforward simulation, as well as higher control resolution in a complex decision process, have been highlighted.

The remainder of this paper is organized as follows. Section 2 introduces some definitions and terminologies, and recaps the simple continuum signal model proposed by Han et al. (2014). Section 3 discusses how the simple continuum model can be modified to accommodate offsets under the no-spillback condition. Section 4 derives the generalized continuum signal model (GCSM) to model networks affected by spillbacks in a very general setting. 


\section{ARTICLE LINK: http://www.sciencedirect.com/science/article/pii/S019126151500048X}

\section{PLEASE CITE THIS ARTICLE AS}

Han, K., Gayah, V.V., 2015. Continuum signalized junction model for dynamic traffic networks: Offset, spillback, and multiple signal phases. Transportation Research Part B 77, 213-239.

Section 5 describes qualitatively how this extended model can be applied to more general and realistic intersection configurations. Numerical examples are provided in Section 6 to validate the proposed model and compare it with the on-off model and the Han et al. (2014) model in a few simulation and optimization tasks. Finally, Section 7 provides a summary of the model extensions and some concluding remarks.

\section{Modeling a signalized intersection: definitions and previous results}

This section provides some definitions and terminologies required to describe the exact on-off signal model, the continuum signal model derived in Han et al. (2014), and the modification/generalization of the continuum model proposed in this paper.

\subsection{Problem setup and terminology}

We assume in this paper that all signals operate with a fixed timing plan. ${ }^{2}$ Specifically, given a fixed time horizon $[0, T]$, and any link $I_{i}$ in the network, where $i=1,2, \ldots$, we define its signal control to be a binary and periodic function $u_{i}(\cdot):[0, T] \rightarrow\{0,1\}$ such that $u_{i}(t)$ equals one if the subject approach receives the green signal, and zero if it receives the red signal. The cycle length, denoted $\Delta_{i}>0$, is equal to the period of $u_{i}(\cdot)$. Throughout this paper, we assume that a signal cycle always starts with the green phase. A fixed split parameter $\eta_{i} \in(0,1)$ is used to describe the green time allocated to the link $I_{i}$; therefore, the actual green time provided to link $I_{i}$ is $\eta_{i} \Delta_{i}$. A central focus of this paper is the signal offset, which is defined as follows.

Definition 2.1. (Signal offset) The offset between two adjacent traffic signals is defined as the difference in the start times of the green period at the downstream signal (say $\left.u_{j}(t)\right)$ and the green period at the upstream signal $\left(\right.$ say $\left.u_{i}(t)\right)$; the offset is denoted $O_{i, j}$.

We now turn to link-specific parameters. It is assumed throughout this paper that the fundamental diagram that describes traffic on all links is triangular, ${ }^{3}$ with the following form:

$$
f(\rho)= \begin{cases}v \rho & \rho \in\left[0, \rho^{c}\right] \\ -w\left(\rho-\rho^{j a m}\right) & \rho \in\left(\rho^{c}, \rho^{j a m}\right]\end{cases}
$$

where $\rho$ denotes density, $v>0$ and $w>0$ are the speeds of the forward- and backward-propagating kinematic waves, respectively; $\rho^{c}$ denotes the critical density at which flow is maximized, and $\rho^{\text {jam }}$ denotes the jam density. We also let $C$ be the flow capacity and $L$ be the link length. All these parameters naturally vary from link to link, and we always stick to the convention of using subscript ' $i$ ' to indicate their dependence on link $I_{i}, i=1,2, \ldots$.

Each link $I_{i}$ is expressed as a spatial interval $\left[a_{i}, b_{i}\right] \subset \mathbb{R}$. The vehicle density is denoted $\rho_{i}(t, x)$ for $(t, x) \in$ $[0, T] \times\left[a_{i}, b_{i}\right]$. The demand $D_{i}(t)$ and supply $S_{i}(t)$ of the link are defined according to Lebacque and Khoshyaran (1999) as:

$$
D_{i}(t)=\left\{\begin{array}{ll}
C_{i} & \text { if } \rho_{i}\left(t, b_{i}-\right) \geq \rho_{i}^{c} \\
f_{i}\left(\rho_{i}\left(t, b_{i}-\right)\right) & \text { if } \rho_{i}\left(t, b_{i}-\right)<\rho_{i}^{c}
\end{array} \quad S_{i}(t)= \begin{cases}C_{i} & \text { if } \rho_{i}\left(t, a_{i}+\right)<\rho_{i}^{c} \\
f_{i}\left(\rho_{i}\left(t, a_{i}+\right)\right) & \text { if } \rho_{i}\left(t, a_{i}+\right) \geq \rho_{i}^{c}\end{cases}\right.
$$

The notion of effective supply is of crucial importance to the definition of signal models.

Definition 2.2. (Effective supply) Given any link $I_{i}$, the effective supply for this link, denoted by $\mathcal{E}_{i}(t)$, is defined as follows. Let $\left\{I_{j}: j=1,2, \ldots, m_{i}\right\}$ be the set of downstream links of $I_{i}$. For each downstream link $I_{j}$, define the car turning percentages $\alpha_{i, j}>0$ such that $\sum_{j=1}^{m_{i}} \alpha_{i, j} \equiv 1$. Then

$$
\mathcal{E}_{i}(t) \doteq \min \left\{C_{i}, \min _{j=1, \ldots, m_{i}}\left\{\frac{S_{j}(t)}{\alpha_{i, j}}\right\}\right\}
$$

The time-varying quantity $\mathcal{E}_{i}(t)$ expresses the downstream receiving capacity available for $I_{i}$ when it receives the green light.

\footnotetext{
${ }^{2}$ This assumption can be relaxed such that the timing plan is allowed to change from one time period (e.g., one cycle) to the next. Here we simply require that the signal timing is fixed during a single cycle.

${ }^{3}$ We note that the results are equally applicable to trapezoidal fundamental diagrams, such as those considered by the cell transmission model.
} 
ARTICLE LINK: http://www.sciencedirect.com/science/article/pii/S019126151500048X

PLEASE CITE THIS ARTICLE AS

Han, K., Gayah, V.V., 2015. Continuum signalized junction model for dynamic traffic networks: Offset, spillback, and multiple signal phases. Transportation Research Part B 77, 213-239.

\subsection{Definition of the on-and-off and continuum signal models}

This section provides a brief review of the on-and-off and continuum models proposed and analyzed in Han et al. (2014). Throughout this paper we will adopt the naming convention of using the superscripts ' $\Delta$ ' and ' 0 ' to represent quantities associated with the on-and-off signal model and the continuum signal model, respectively. Using the signal control $u_{i}(t)$ and effective supply $\mathcal{E}_{i}(t)$ for link $I_{i}$, the on-and-off model for this link is expressed in terms of its downstream boundary condition:

$$
f_{\text {out }, i}^{\Delta}(t)=\min \left\{D_{i}(t), u_{i}(t) \mathcal{E}_{i}(t)\right\} \quad \text { (On-and-off signal model) }
$$

where $f_{\text {out }, i}^{\Delta}(t)$ denotes the exit flow of $I_{i}$. On the other hand, the continuum signal model is defined as:

$$
f_{\text {out }, i}^{0}(t)=\min \left\{D_{i}(t), \eta_{i} \mathcal{E}_{i}(t)\right\} \quad \text { (Continuum signal model) }
$$

Remark 2.3. The difference between the on-and-off model and the continuum model lies in the way downstream boundary conditions are specified. A general network flow problem also involves initial and upstream boundary conditions, which are implicitly considered by this paper. In other words, all the results of this paper hold true with any given initial and upstream boundary conditions. The reader is referred to Han et al. (2014) for a LaxHopf treatment of various conditions in conjunction with the Hamilton-Jacobi equation and the Moskowitz function (Moskowitz, 1965), which serves as the foundation of our analysis in this paper.

The following table summarizes the key variables that will be used in this paper.

$f_{i n, i}^{\Delta}(t) \quad$ the inflow of link $I_{i}$ with the on-and-off model

$f_{i n, i}^{0}(t) \quad$ the inflow of link $I_{i}$ with the continuum model

$f_{\text {out }, i}^{\Delta}(t) \quad$ the exit flow of link $I_{i}$ with the on-and-off model

$f_{\text {out }, i}^{0}(t) \quad$ the exit flow of link $I_{i}$ with the continuum model

$N_{u p, i}^{\Delta}(t) \quad$ the cumulative entering vehicle count of link $I_{i}$ with the on-and-off model

$N_{u p, i}^{0}(t) \quad$ the cumulative entering vehicle count of link $I_{i}$ with the continuum model

$N_{d o w n, i}^{\Delta}(t)$ the cumulative exiting vehicle count of link $I_{i}$ with the on-and-off model

$N_{\text {down, } i}^{0}(t)$ the cumulative exiting vehicle counts of link $I_{i}$ with the continuum model

$N_{i}^{\Delta}(t, x) \quad$ the Moskowitz function of link $I_{i}$ with the on-and-off model

$N_{i}^{0}(t, x) \quad$ the Moskowitz function of link $I_{i}$ with the continuum model

$D_{i}(t) \quad$ the demand of link $I_{i}$

$S_{i}(t) \quad$ the supply of link $I_{i}$

$\mathcal{E}_{i}(t) \quad$ the effective supply for link $I_{i}$

$u_{i}(t) \quad$ the signal control for $I_{i}$

$\Delta_{i} \quad$ the cycle length of the signal control $u_{i}(t)$

$\eta_{i} \quad$ the green split for link $I_{i}$

$O_{i, j} \quad$ the offset between two adjacent signal controls $u_{i}(t)$ and $u_{j}(t)$

\section{Continuum signal model considering offset: the no-spillback case}

We extend the continuum signal model proposed in Han et al. (2014) to explicitly capture signal offsets along a signalized corridor. Without loss of generality, we consider the corridor depicted in Figure 1. 


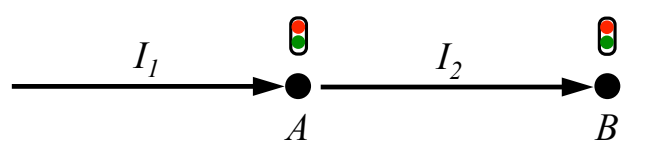

Figure 1: A signalized corridor with coordinated signals at junctions $A$ and $B$.

The following main assumptions will be made in this section.

(A1). No spillback occurs along the corridor. In other words, the supply $S_{i}(t)$ of each link is a constant and equal to $C_{i}$, the flow capacity.

(A2). All links have the same cycle time and maximum throughput per cycle $\mathcal{M}_{i}$, which is defined to be the product of the green time and the effective supply, i.e. $\mathcal{M}_{i}=\eta_{i} \Delta_{i} \mathcal{E}_{i}$.

The case with spillback will be handled in the next section.

In order to describe the flow propagation in the absence of vehicle spillback, we employ the following variational formulation based on Newell (1993) and Han et al. (2013a) and the assumption that no spillback occurs (a mathematical derivation of the following formulae will be presented in Appendix A).

$$
\begin{aligned}
& N_{\text {down }, i}^{\Delta}(t)=\min _{\tau \leq t}\left\{N_{u p, i}^{\Delta}\left(\tau-\mathcal{T}_{i}\right)-V_{i}^{\Delta}(\tau)\right\}+V_{i}^{\Delta}(t) \quad \text { (on-and-off) } \\
& N_{\text {down }, i}^{0}(t)=\min _{\tau \leq t}\left\{N_{u p, i}^{0}\left(\tau-\mathcal{T}_{i}\right)-V_{i}^{0}(\tau)\right\}+V_{i}^{0}(t) \quad \text { (continuum) }
\end{aligned}
$$

where $\mathcal{T}_{i}$ denotes the free-flow time on link $I_{i}$; and

$$
V_{i}^{\Delta}(t) \doteq \int_{0}^{t} u_{i}(\tau) \cdot \mathcal{E}_{i} d \tau, \quad V_{i}^{0}(t) \doteq \int_{0}^{t} \eta_{i} \cdot \mathcal{E}_{i}(\tau) d \tau=\eta_{i} \mathcal{E}_{i} t
$$

are the weak downstream boundary conditions defined to be the anti-derivatives of the respective downstream capacity with on-and-off/continuum signal controls incorporated. Notice that in view of (A1), the effective supply $\mathcal{E}_{i}(\cdot)$ is equal to a constant, $i=1,2$. We make note of the following useful fact.

[Fact 1]. Adding a constant to the function $V_{i}^{\Delta}(\cdot)\left(\right.$ or $\left.V_{i}^{0}(\cdot)\right)$ does not affect the function $N_{\text {down }, i}^{\Delta}(\cdot)\left(\right.$ or $\left.N_{\text {down,i }}^{0}(\cdot)\right)$. This is obvious from (3.6) and (3.7).

\subsection{Theoretical error estimation and model development}

In view of Figure 1, we use the variational formulation (3.6)-(3.7) and knowledge of the signal offset to estimate the error associated with the continuum model (2.5). The following additional set of notations are recapped.

$$
\begin{array}{ll}
V_{i}^{\Delta}(t) & \text { the weak downstream boundary condition of } I_{i} \text { with the on-and-off model } \\
V_{i}^{0}(t) & \text { the weak downstream boundary condition of } I_{i} \text { with the continuum model } \\
\mathcal{T}_{i} & \text { the free-flow time on link } I_{i} \\
\mathcal{M}_{i} & \text { the maximum throughput per cycle of link } I_{i} \text { (defined in (A2)) }
\end{array}
$$

Next, we define the quantity $\delta(t)$, which is closely related to the relative phase of the two signals $u_{1}(t)$ and $u_{2}(t)$, and turns out to be the key to the continuum approximation error when offset is considered. Without loss of generality, we let $t=0$ be the start of the cycle (and the green phase) of $u_{1}(\cdot)$. Then, with the offset $O_{1,2}$, we can explicitly write

$$
u_{1}(t)=\left\{\begin{array}{ll}
1 & \left\langle\frac{t}{\Delta}\right\rangle \leq \eta_{1} \\
0 & \left\langle\frac{t}{\Delta}\right\rangle>\eta_{1}
\end{array} \quad u_{2}(t)= \begin{cases}1 & \left\langle\frac{t-O_{1,2}}{\Delta}\right\rangle \leq \eta_{2} \\
0 & \left\langle\frac{t-O_{1,2}}{\Delta}\right\rangle>\eta_{2}\end{cases}\right.
$$

where $\langle x\rangle$ represents the decimal part of a real number $x$, and $\Delta$ is the common cycle time of the two signals as stipulated by (A2). We are now ready to define the function $\delta(t)$. 
ARTICLE LINK: http://www.sciencedirect.com/science/article/pii/S019126151500048X

PLEASE CITE THIS ARTICLE AS

Han, K., Gayah, V.V., 2015. Continuum signalized junction model for dynamic traffic networks: Offset, spillback, and multiple signal phases. Transportation Research Part B 77, 213-239.

Definition 3.1. Given any signal cycle $\Delta$, splits $\eta_{1}, \eta_{2}$, and offset $O_{1,2}$, we have

$$
V_{1}^{\Delta}\left(t-\mathcal{T}_{2}\right)=\int_{0}^{t-\mathcal{T}_{2}} u_{1}(\tau) \mathcal{E}_{1} d \tau, \quad V_{2}^{\Delta}(t)=\int_{0}^{t} u_{2}(\tau) \mathcal{E}_{2} d \tau
$$

where $u_{1}(\cdot)$ and $u_{2}(\cdot)$ are given by (3.8). The difference $\left|V_{1}^{\Delta}\left(t-\mathcal{T}_{2}\right)-V_{2}^{\Delta}(t)\right|$ is uniformly bounded due to (A2). We then revise the function $V_{1}^{\Delta}\left(t-\mathcal{T}_{2}\right)$ by subtracting from it a constant $\min _{t \in[0, T]}\left\{V_{1}^{\Delta}\left(t-\mathcal{T}_{2}\right)-V_{2}^{\Delta}(t)\right\}$, i.e.

$$
V_{1}^{\Delta}\left(t-\mathcal{T}_{2}\right) \longleftarrow V_{1}^{\Delta}\left(t-\mathcal{T}_{2}\right)-\min _{t \in[0, T]}\left\{V_{1}^{\Delta}\left(t-\mathcal{T}_{2}\right)-V_{2}^{\Delta}(t)\right\}
$$

without affecting the model outcome by virtue of Fact 1. The adjustment (3.9) will be adopted throughout the rest of this section. Accordingly, we define

$$
\delta(t) \doteq V_{1}^{\Delta}\left(t-\mathcal{T}_{2}\right)-V_{2}^{\Delta}(t)
$$

which can be easily shown to be non-negative and uniformly bounded, where $V_{1}^{\Delta}\left(t-\mathcal{T}_{2}\right)$ has been adjusted according to (3.9).

According to Definition 3.1, $\delta(\cdot)$ is determined entirely by $\Delta, \eta_{1}, \eta_{2}, \mathcal{T}_{2}, O_{1,2}$ and nothing else. The next theorem estimates the continuum approximation error taking into account the effect of the offset, which is encapsulated in $\delta(t)$.

Theorem 3.2. (Error estimate considering offset) Consider the signalized corridor in Figure 1. Let assumptions (A1)-(A2) hold. Applying the continuum model (2.5) to both links yields the following error estimates.

$$
-\eta_{1}\left(1-\eta_{1}\right) \Delta \mathcal{E}_{1} \leq N_{\text {down,2 }}^{0}(t)-N_{d o w n, 2}^{\Delta}(t) \leq \eta_{1}\left(1-\eta_{1}\right) \Delta \mathcal{E}_{1}+\delta(t)
$$

where $\eta_{1}$ and $\mathcal{E}_{1}=\min \left\{C_{1}, C_{2}\right\}$ are the green split and the (constant) effectively supply for link $I_{1}$, respectively; $\Delta$ is the common signal cycle for both links.

Proof. The proof is postponed until Appendix B.1.

Remark 3.3. Han et al. (2014) provide the following error estimate for the single link $I_{1}$ :

$$
\left|N_{\text {down, } 1}^{0}(t)-N_{\text {down, } 1}^{\Delta}(t)\right| \leq \eta_{1}\left(1-\eta_{1}\right) \Delta \mathcal{E}_{1}
$$

and predict that the error for the next link $I_{2},\left|N_{\text {down,2 }}^{0}(t)-N_{\text {down,2 }}^{\Delta}(t)\right|$, may pick up an additional error and be as large as $\eta_{1}\left(1-\eta_{1}\right) \Delta \mathcal{E}_{1}+\eta_{2}\left(1-\eta_{2}\right) \Delta \mathcal{E}_{2}$ as the continuum approximation error propagates to the next link $I_{2}$. The significance of (3.11) is that it provides a much sharper error estimate, and attributes the additional error to the function $\delta(t)$, which depends on the offset.

Eqn (3.11) leads to the following time-invariant error bounds

$$
-\eta_{1}\left(1-\eta_{1}\right) \Delta \mathcal{E}_{1} \leq N_{d o w n, 2}^{0}(t)-N_{d o w n, 2}^{\Delta}(t) \leq \eta_{1}\left(1-\eta_{1}\right) \Delta \mathcal{E}_{1}+\max _{t \in[0, T]} \delta(t)
$$

Notice that the lower and upper bounds in (3.13) are not symmetric, and the absolute error between $N_{d o w n, 2}^{0}(t)$ and $N_{\text {down,2 }}^{\Delta}(t)$ is bounded by $\eta_{1}\left(1-\eta_{1}\right) \Delta \mathcal{E}_{1}+\max _{t \in[0, T]} \delta(t)$. We propose the following modification of the continuum model.

Definition 3.4. (Modified continuum model in the absence of spillback) Given signal controls $u_{1}(\cdot)$ and $u_{2}(\cdot)$, we define $\delta(\cdot)$ according to Definition 3.1. Then the new continuum signal model considering signal offset is defined as

$$
\hat{N}_{d o w n, 2}^{0}(t) \doteq N_{d o w n, 2}^{0}(t)-\frac{1}{2} \max _{t \in[0, T]} \delta(t)
$$

where $N_{d o w n, 2}^{0}(t)$ is the result of the original continuum model (2.5) of Han et al. (2014). 
ARTICLE LINK: http://www.sciencedirect.com/science/article/pii/S019126151500048X

PLEASE CITE THIS ARTICLE AS

Han, K., Gayah, V.V., 2015. Continuum signalized junction model for dynamic traffic networks: Offset, spillback, and multiple signal phases. Transportation Research Part B 77, 213-239.

It can be seen that the new model yields symmetric upper and lower bounds; that is,

$$
-\eta_{1}\left(1-\eta_{1}\right) \Delta \mathcal{E}_{1}-\frac{1}{2} \max _{t \in[0, T]} \delta(t) \leq \hat{N}_{\text {down, }}^{0}(t)-N_{d o w n, 2}^{\Delta}(t) \leq \eta_{1}\left(1-\eta_{1}\right) \Delta \mathcal{E}_{1}+\frac{1}{2} \max _{t \in[0, T]} \delta(t)
$$

The improvement of this new continuum model can be seen from the following comparison:

$$
\begin{aligned}
& \left|N_{\text {down, },}^{0}(t)-N_{\text {down, } 2}^{\Delta}(t)\right| \leq \eta_{1}\left(1-\eta_{1}\right) \Delta \mathcal{E}_{1}+\max _{t \in[0, T]} \delta(t) \\
& \left|\hat{N}_{\text {down, } 2}^{0}(t)-N_{\text {down, } 2}^{\Delta}(t)\right| \leq \eta_{1}\left(1-\eta_{1}\right) \Delta \mathcal{E}_{1}+\frac{1}{2} \max _{t \in[0, T]} \delta(t)
\end{aligned}
$$

which implies a reduction of error by $\frac{1}{2} \max _{t \in[0, T]} \delta(t)$. In the next subsection we will illustrate and quantify $\delta(t)$ as well as the improvement of the modified continuum model, when a range of offsets are considered.

\subsection{Examples}

To fully understand the impact of the offset on the function $\delta(\cdot)$, we use Figure 2 to depict a number of possible configurations of $V_{1}^{\Delta}\left(t-\mathcal{T}_{2}\right)$ and $V_{2}^{\Delta}(\tau)$, along with $\delta(\tau)$. We will go through these cases and illustrate the magnitude of $\delta(t)$ and its relationship with the offset. We consider a simplified case where $C_{1}=C_{2}=\mathcal{E}_{1}=\mathcal{E}_{2}=C, \eta_{1}=\eta_{2}=\eta$, and $\eta \leq \frac{1}{2}$. Notice that these can be easily relaxed and the result is applicable to more general cases. We use $O_{1,2}$ to denote the offset between $u_{1}(t)$ and $u_{2}(t)$.

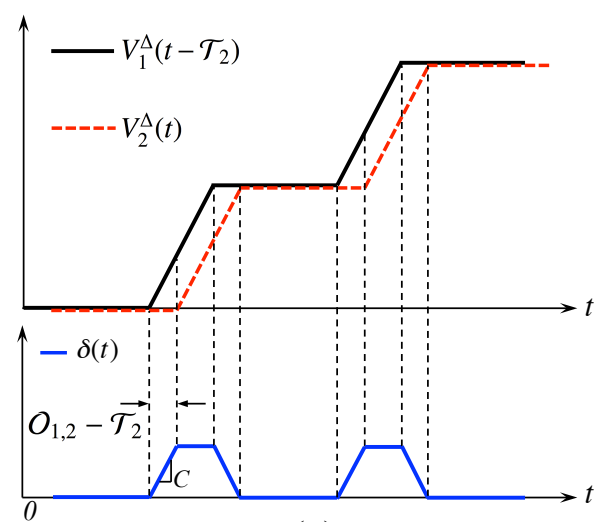

(a)

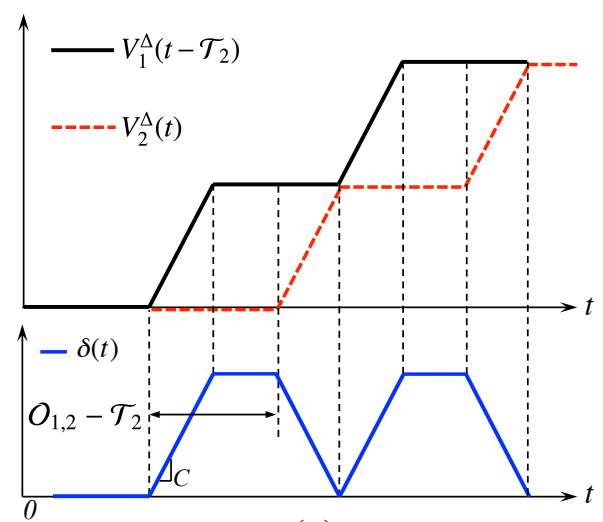

(c)

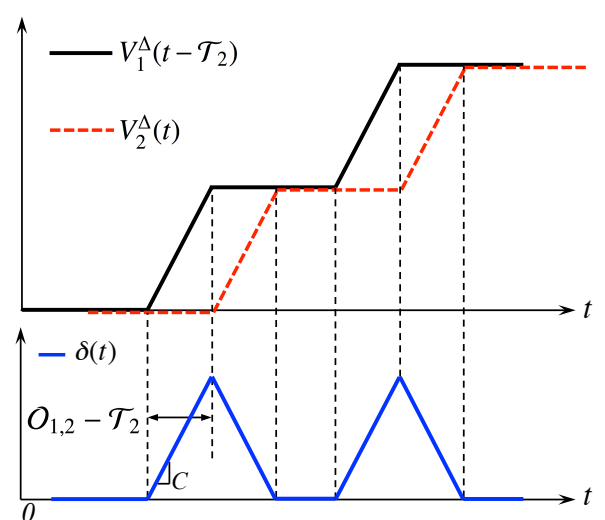

(b)

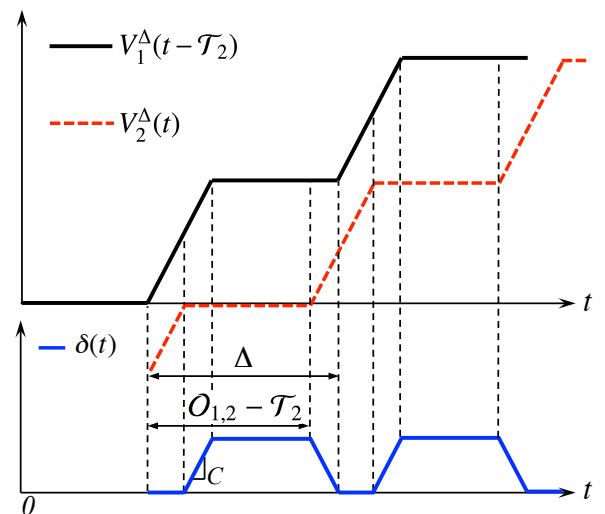

(d)

Figure 2: Four illustrative scenarios of the configuration of $V_{1}^{\Delta}\left(\tau-\mathcal{T}_{2}\right)$ and $V_{2}^{\Delta}(\tau)$. 


\section{ARTICLE LINK: http://www.sciencedirect.com/science/article/pii/S019126151500048X}

\section{PLEASE CITE THIS ARTICLE AS}

Han, K., Gayah, V.V., 2015. Continuum signalized junction model for dynamic traffic networks: Offset, spillback, and multiple signal phases. Transportation Research Part B 77, 213-239.

- Case (a): $O_{1,2}-\mathcal{T}_{2} \in(0, \eta \Delta]$. In Figure 2(a), the quantity $O_{1,2}-\mathcal{T}_{2}$ shown in the picture is relatively small. The maximum of the periodic function $\delta(t)$ is $C \cdot\left(O_{1,2}-\mathcal{T}_{2}\right)$.

- Case (b) and (c): $O_{1,2}-\mathcal{T}_{2} \in(\eta \Delta,(1-\eta) \Delta]$. In this case, the magnitude of $\delta(t)$ reaches its theoretical maximum: $\eta \Delta C$. Moreover, the error is more severe in case (c) than case (b) as the maximum is sustained for longer periods, as can be seen from the plateaus in the $\delta(\cdot)$ function in case (c).

- Case (d): $O_{1,2}-\mathcal{T}_{2} \in((1-\eta) \Delta, \Delta]$. In this case $\min _{t \in[0, T]}\left\{V_{1}^{\Delta}\left(t-\mathcal{T}_{2}\right)-V_{2}^{\Delta}(t)\right\}$ is greater than zero. Following the adjustment proposed in (3.9) by subtracting this positive constant from $V_{1}^{\Delta}\left(t-\mathcal{T}_{2}\right)$, we obtain the non-negative function $\delta(t)$ whose maximum is given as $C\left(\Delta-\left(O_{1,2}-\mathcal{T}_{2}\right)\right)$; see Figure 2(d). Finally, when $O_{1,2}-\mathcal{T}_{2}=\Delta$, the function $\delta(t) \equiv 0$.

Let us summarize the general trend of the function $\delta(\cdot)$ when the quantity $O_{1,2}-\mathcal{T}_{2}$ varies from 0 to $\Delta$. When $O_{1,2}-\mathcal{T}_{2}$ increases from 0 to $\eta \Delta$ (case (a)), the error increases gradually from 0 to its maximum: $\eta \Delta C$. As $O_{1,2}-\mathcal{T}_{2}$ keeps increasing from $\eta \Delta$ to $(1-\eta) \Delta$ (cases (b) and (c)), the maximum error remains the same, but is maintained for longer periods as $O_{1,2}-\mathcal{T}_{2}$ increases. Once $O_{1,2}-\mathcal{T}_{2}$ exceeds $(1-\eta) \Delta$ (case $(\mathrm{d})$ ), the magnitude of the error begins to decrease, and returns to zero when $O_{1,2}-\mathcal{T}_{2}$ reaches $\Delta$.

We note that the error function $\delta(t)$ is identically zero when $O_{1,2}=\mathcal{T}_{2}$; that is, when the offset is equal to the free-flow time on the link $I_{2}$.

\subsection{Numerical validation}

This section validates our theoretical results developed so far on the continuum approximation error. We consider again the corridor shown in Figure 1. The signal parameters are: $\Delta=60 \mathrm{~s}, \eta_{1}=\eta_{2}=1 / 3$, and the flow capacities are $C_{1}=C_{2}=4 / 3 \mathrm{veh} / \mathrm{s}$. For the on-and-off model, 12 different signal offsets are considered: $0,5,10, \ldots, 55 \mathrm{~s}$. For the continuum model, we compare the Han et al. (2014) model (2.5) and the proposed model (3.14) in terms of their approximation accuracy.

In the presentation of the numerical results, we select $O_{1,2}=\mathcal{T}_{2}$ as the base reference and consider offsets that deviate from $\mathcal{T}_{2}$ by $+5,+10, \ldots,+55$. For each chosen signal offset, 1000 simulation runs were conducted with random inflows of link $I_{1}$ generated between zero and the flow capacity. The following performance measures are considered and summarized in Figure 3(i) and Figure 3(ii), respectively.

$$
\max _{t \in[0, T]}\left\{\left|N_{\text {down,2 }}^{0}(t)-N_{\text {down,2 }}^{\Delta}(t)\right|\right\} \quad \text { and } \quad \max _{t \in[0, T]}\left\{\left|\hat{N}_{\text {down,2 }}^{0}(t)-N_{\text {down, }}^{\Delta}(t)\right|\right\}
$$

Figure 3(i) shows remarkable consistency between the error in addition to $\eta(1-\eta) \Delta C$, caused by the signal offset, and the description of the function $\delta(\cdot)$ in Section 3.2. Indeed, as the offset deviate from the free-flow time $\mathcal{T}_{2}$, the error increases as predicted throughout cases (a), (b) and (c). Past the point $(1-\eta) \Delta=40$ s, the error begins to decrease, as predicted in case (d). When the offset equals the free-flow time, the error remains bounded by $\eta(1-\eta) \Delta C=17.78$ (vehicles), which coincides with the error bound for a single link. In addition, the maximum possible error is bounded by twice the error for a single link case $(2 \eta(1-\eta) \Delta C)$, which is consistent with the observation made by Han et al. (2014): The continuum approximation error may add up when traffic propagates from one link to another. This is, however, the worse case scenario and it is attained precisely at the "worst offset" (equal to the free-flow time plus 40 s). On the other hand, it can be seen from Figure 3(ii) that the new model proposed in this paper, having taken into account the offset effect, substantially reduces the error, and maintains the error almost within the error bound of a single link case, that is, $\eta(1-\eta) \Delta C=17.78$ vehicles.

\section{Generalized continuum signal model (GCSM) for the spillback case}

As far as continuum signal approximation is concerned, the key difference in the spillback and no-spillback cases, and a significant source of theoretical difficulty, lie in the fact that the supply of the downstream link is no longer constant when spillback is present. Thus, the discharge flow of an upstream link is jointly determined by the signal timing and the downstream supply profile, which, in turn, may depend on the discharge flows of upstream links. In this section, we propose a generalized continuum signal model (GCSM), extending the Han et al. (2014) model to properly handle vehicle spillback from downstream links. 
ARTICLE LINK: http://www.sciencedirect.com/science/article/pii/S019126151500048X

PLEASE CITE THIS ARTICLE AS

Han, K., Gayah, V.V., 2015. Continuum signalized junction model for dynamic traffic networks: Offset, spillback, and multiple signal phases. Transportation Research Part B 77, 213-239.

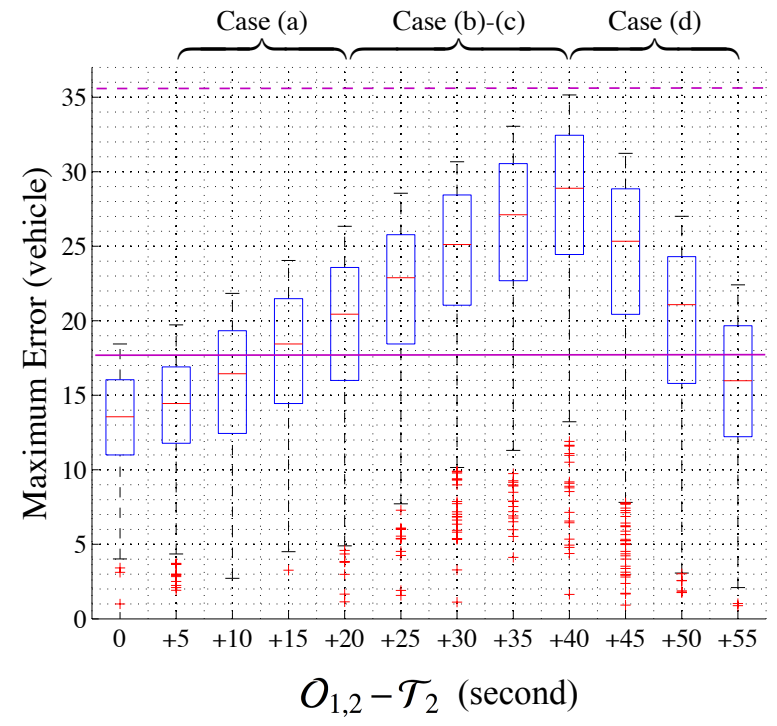

(i)

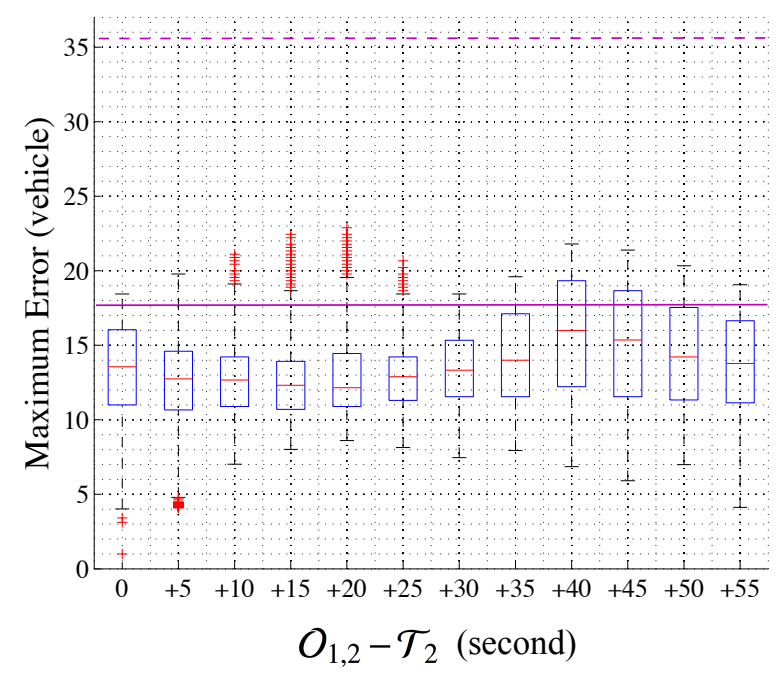

(ii)

Figure 3: Box plots of the maximum absolute error for the (Han et al., 2014) model (left), and the proposed continuum model (right).

\subsection{Some examples of spillback}

To properly motivate the GCSM and to facilitate our presentation in subsequent subsections, we provide here some intuitive examples to illustrate the spillback phenomena on a signalized network. In particular, we consider the network depicted in Figure 4. The following relationship among some parameters are assumed to generate spillback: $\Delta_{5}=2 \Delta_{4}, C_{5}<C_{4}, C_{5}<C_{7}$.

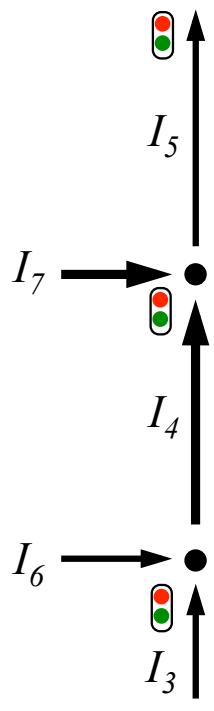

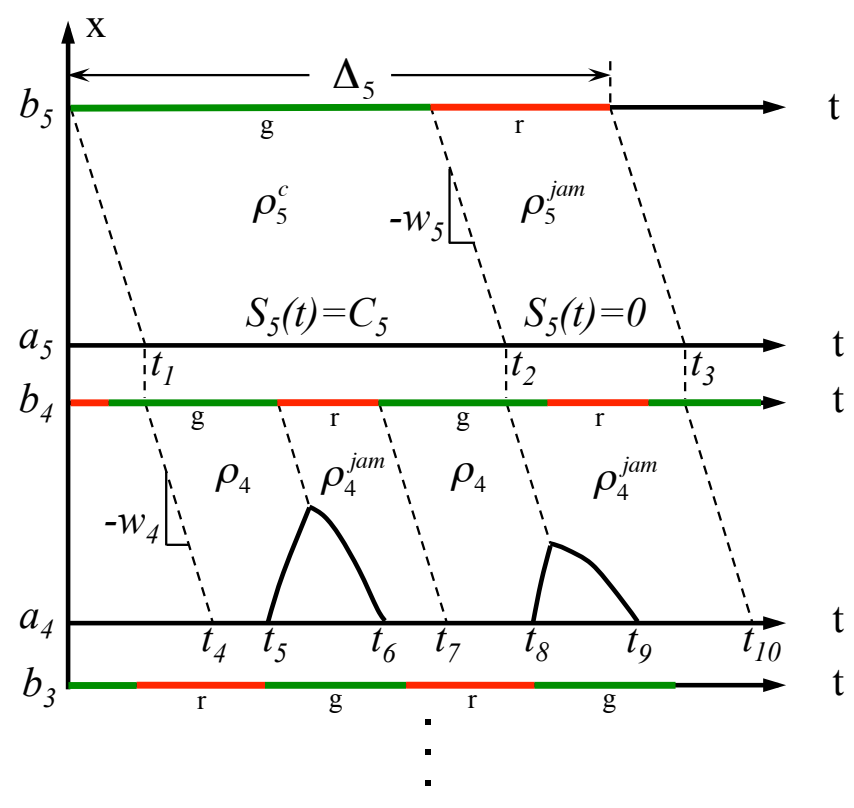

Figure 4: An illustration of spillback. 

and multiple signal phases. Transportation Research Part B 77, 213-239.

Figure 4 shows the space-time diagrams for $I_{4}$ and $I_{5}$ when spillback is triggered by $I_{5}$ and affects the two upstream nodes; and we assume that the downstream of $I_{5}$ is free of congestion. At the downstream end of $I_{5}\left(x=b_{5}\right)$ the signal $u_{5}(t)$ generates two density values: $\rho_{5}^{c}$ (green phase) and $\rho_{5}^{\text {jam }}$ (red phase); both densities propagate at the same speed $-w_{5}$. Assume both $I_{4}$ and $I_{7}$ discharge vehicles into $I_{5}$ at the maximum rate $C_{5}$ during their respective green phases (recall that $\left.C_{4}, C_{7}>C_{5}\right)$, then the upstream boundary of $I_{5}\left(x=a_{5}\right)$ remains congested during an entire cycle $\Delta_{5}$. We will call this situation sustained spillback. Consequently, the supply function of $I_{5}$ is explicitly written as

$$
S_{5}(t)= \begin{cases}C_{5} & t \in\left[t_{1}, t_{2}\right] \\ 0 & t \in\left(t_{2}, t_{3}\right]\end{cases}
$$

We now turn to link $I_{4}$. Notice that due to the congestion on $I_{5}$, cars can be discharged from $I_{4}$ only if $u_{4}(t)=1$ and $S_{5}(t)=C_{5}$, which highlights the key difference from the no-spillback case. Specifically, we have

$$
f_{\text {out }, 4}(t)= \begin{cases}C_{5} & \text { if } u_{4}(t) \cdot S_{5}(t)>0 \\ 0 & \text { otherwise }\end{cases}
$$

When $f_{\text {out }, 4}(t)=C_{5}$, the corresponding density value, denoted $\rho_{4}$, satisfies $\rho_{4}^{c}<\rho_{4}<\rho_{4}^{j a m}$ and $f_{4}\left(\rho_{4}\right)=C_{5}$. When $f_{\text {out }, 4}(t)=0$, the corresponding density is $\rho_{4}^{\text {jam }}$. Both density values propagate at the speed of $-w_{4}$. At the upstream boundary of $I_{4}\left(x=a_{4}\right)$, if the inflow $f_{i n, 4}(t)$ is less than $C_{4}$, a shock wave will emerge (say at time $\left.t_{5}\right)$ and propagates forward into the link. After the shock intersects the density $\rho_{4}^{\text {jam }}$, it will propagate backward and return to the boundary $x=a_{4}$ (say at $t_{6}$ ). As a consequence, for the scenario depicted in Figure 4, we have

$$
S_{4}(t)= \begin{cases}C_{5} & t \in\left[t_{4}, t_{5}\right] \cup\left[t_{7}, t_{8}\right] \\ C_{4} & t \in\left(t_{5}, t_{6}\right] \cup\left(t_{8}, t_{9}\right] \\ 0 & t \in\left(t_{6}, t_{7}\right) \cup\left(t_{9}, t_{10}\right]\end{cases}
$$

In this case, the traffic at $x=a_{4}$ may switch between the free-flow and congested phases. And we call such a situation transient spillback, as the spillback may recede for some time.

The dynamic pattern illustrated in Figure 4 is expected to repeat every $\Delta_{5}$ units of time, and the supplies $S_{5}(\cdot)$ and $S_{4}(\cdot)$ are periodic with period $\Delta_{5}$ (more generally, the period is the least common multiple of $\Delta_{5}$ and $\Delta_{4}$ but since $\Delta_{5}=2 \Delta_{4}$ the LCM is just $\Delta_{5}$ ). While the scenario depicted in Figure 4 is only illustrative, the following observations hold true on a more general ground.

1. Both sustained and transient spillbacks may take place, depending on the junction configuration and upstream flow profiles.

2. When spillback occurs in a network, the supply function of a link, and the effective supply functions of its upstream links, may be piecewise constant functions taking two or more values.

\subsection{The generalized continuum signal model (GCSM)}

The previous example reveals complex behavior at junctions affected by the spillback. In order to unify a wide range of scenarios and analyze the continuum approximation in the most general setting, we propose a generalized continuum signal model (GCSM). Let us focus on an arbitrary link in a given network. For simplicity of notation, the subscript $i$ indicating link ID will be omitted in this subsection. The following assumption, motivated by the observations made in Section 4.1, is employed by the GCSM.

(A3). The effective supply function $\mathcal{E}(\cdot)$ for the link of interest is a piecewise constant function. Moreover, $\mathcal{E}(\cdot)$ is periodic with period denoted by $\Lambda$.

Remark 4.1. (A3) is a very general assumption which will be justified later for a variety of spillback scenarios. It holds true even in the no-spillback case, in which case $\mathcal{E}(\cdot)$ is a constant and, thus, a periodic function with period $\Lambda$ arbitrarily defined.

One key notion of the GCSM is the effective signal control (ESC), which is a generalization of the real-world signal control taking into consideration possible downstream blockage effect caused by spillback. 
ARTICLE LINK: http://www.sciencedirect.com/science/article/pii/S019126151500048X

PLEASE CITE THIS ARTICLE AS

Han, K., Gayah, V.V., 2015. Continuum signalized junction model for dynamic traffic networks: Offset, spillback, and multiple signal phases. Transportation Research Part B 77, 213-239.

Definition 4.2. (Effective signal control (ESC)) Under assumption (A3), the effective signal control (ESC) for the subject link, denoted $\mathbf{u}(\cdot)$, is defined as

$$
\mathbf{u}(t) \doteq \begin{cases}1 & \text { if } u(t) \cdot \mathcal{E}(t)>0 \\ 0 & \text { if } u(t) \cdot \mathcal{E}(t)=0\end{cases}
$$

where $u(\cdot)$ is the real-world signal control. Obviously $\mathbf{u}(\cdot)$ is a periodic function, and we call the "green phase" of $\mathbf{u}(\cdot)$ the discharge window $(D W)$.

Remark 4.3. According to Definition 4.2, vehicles can be discharged from the subject link at a positive rate during the discharge window. Outside the DW cars cannot leave this link due to: (1) the red light $(u(t)=0)$; or, (2) zero downstream receiving capacity $(\mathcal{E}(t)=0)$. If there were no blockage effect (spillback) from downstream links, then the ESC coincides with the real-world signal control. However, in the presence of downstream spillback the discharge flow may be zero even if the light is green. Consequently, we need to resort to the more general ESC to handle such a situation. In general, $\mathbf{u}(\cdot)$ may have several green and red phases in a full cycle. An example of this is illustrated previously in Figure 4.

Definition 4.4. Under assumption (A3), given the real-world signal control $u(\cdot)$ with cycle length $\Delta$, and the effective supply $\mathcal{E}(\cdot)$ with period $\Lambda$, we define

$$
\Omega \doteq \operatorname{LCM}(\Delta, \Lambda)
$$

where LCM stands for least common multiple. $\Omega$ is equal to the period of the function $u(\cdot) \cdot \mathcal{E}(\cdot)$.

The following generalized continuum signal model arises from the observation that the exit flow of the subject link is determined by $u(\cdot) \cdot \mathcal{E}(\cdot)$, which has a period of $\Omega$.

Definition 4.5. (Generalized continuum signal model (GCSM)) Under assumption (A3), the generalized continuum signal model is defined via the following downstream boundary condition:

$$
f_{\text {out }}^{0}(t)=\min \{D(t), R\}
$$

where $D(\cdot)$ is the demand function defined in (2.2), and $R$ is defined as

$$
R \doteq \frac{1}{\Omega} \int_{t_{0}}^{t_{0}+\Omega} u(t) \cdot \mathcal{E}(t) d t
$$

where $t_{0}$ is arbitrary.

Theorem 4.6. (Error estimate for the GCSM) Let assumption (A3) hold, with the piecewise constant function $u(\cdot) \cdot \mathcal{E}(\cdot)$ taking values in $\left\{K_{0}, K_{1}, \ldots, K_{m}\right\}$ where $0=K_{0}<K_{1} \ldots<K_{m} \leq C$. Given the same initial and upstream boundary conditions, the two Moskowitz functions, corresponding to the on-and-off model (2.4) and the GCSM (4.20) respectively, satisfy

$$
\left|N^{0}(t, x)-N^{\Delta}(t, x)\right| \leq \sum_{j: K_{j} \leq R} \mu\left(\beta_{j}\right)\left(R-K_{j}\right)
$$

where $\beta_{j} \doteq\left\{t \in[0, \Omega]: u(t) \cdot \mathcal{E}(t)=K_{j}\right\}, 0 \leq j \leq m, \mu(\cdot)$ is the Lebesgue measure. To simply put, for every $0 \leq j \leq m$, $\mu\left(\beta_{j}\right)$ is the time duration within a cycle of $u(\cdot) \cdot \mathcal{E}(\cdot)$ when $u(t) \cdot \mathcal{E}(t)=K_{j}$.

Proof. The proof is postponed until Appendix B.2.

Remark 4.7. One immediately recognizes the continuum model (2.5) without spillback as a special case of (4.20), by setting the effective supply $\mathcal{E}(t)$ to be a constant $\overline{\mathcal{E}}$, and thus reducing (4.21) to $\eta \overline{\mathcal{E}}$, where $\eta$ is the green split of the real-world signal. In terms of error bound (4.22), in the absence of spillback, the image of $u(\cdot) \cdot \mathcal{E}(\cdot)$ reduces to $\{0, \overline{\mathcal{E}}\}$; and $\mu\left(\beta_{0}\right)=(1-\eta) \Delta, \mu\left(\beta_{1}\right)=\eta \Delta$. Thus, the right hand side of (4.22) becomes

$$
\mu\left(\beta_{0}\right)(\eta \overline{\mathcal{E}}-0)=\eta(1-\eta) \Delta \overline{\mathcal{E}}
$$

which is exactly the error bound for the no-spillback case (Han et al., 2014). Therefore, the GCSM unifies both the no-spillback and spillback cases through the notion of effective signal control and effective supply. 
ARTICLE LINK: http://www.sciencedirect.com/science/article/pii/S019126151500048X

PLEASE CITE THIS ARTICLE AS

Han, K., Gayah, V.V., 2015. Continuum signalized junction model for dynamic traffic networks: Offset, spillback, and multiple signal phases. Transportation Research Part B 77, 213-239.

In order to apply the GCSM to handle various spillback scenarios, one crucial step is to verify assumption (A3) and specify $\mathcal{E}(\cdot)$ in conjunction with the intersection type and upstream and/or downstream flow profiles. This will be achieved in the sections below. The following newly introduced notations are emphasized.
$\mathbf{u}_{i}(t)$
the effective signal control (ESC) for link $I_{i}$;
$\Omega_{i} \quad$ the period of the function $u_{i}(\cdot) \cdot \mathcal{E}_{i}(\cdot)$, as defined in (4.19), for link $I_{i}$.

\subsection{Estimating link supply in the presence of spillback}

As we have previously seen that, in order to apply the GCSM, it is crucial to estimate the downstream supply profile while taking into account its interaction and coupling with the upstream signal controls and links. To this end, we introduce two methods for calculating the supply $S(\cdot)$ of a link given its signal control $u(\cdot)$ and effective supply $\mathcal{E}(\cdot)$. Obviously, if a link does not trigger spillback then its supply is equal to the flow capacity. Thus, for what follows it is always assumed that the subject link is congested and triggers spillback to its upstream node. In application, the presence of spillback can always be verified using a simple if-then statement following the variational principle, and an example of this is presented at the end of this section, in Algorithm III.

\subsubsection{Method I for estimating supply $S(\cdot)$ given $u(\cdot)$ and $\mathcal{E}(\cdot)$}

The first method is based on the following assumption (the subscript index ' $i$ ' is omitted for the subject link here):

(A4). $\mathcal{E}(t) \in\{0, C\}$.

Remark 4.8. Either one of the following statements is equivalent to (A4):

(1) $u(t) \cdot \mathcal{E}(t)=C \mathbf{u}(t)$.

(2) Cars can be discharged from the subject link at the flow capacity $C$ whenever $\mathbf{u}(t)=1$.

Moreover, a sufficient condition for (A4) to hold is that the link flow capacity is non-increasing in the direction of spillback (or the opposite direction of travel). In particular, (A4) holds for corridors where all links have the same flow capacity. Notice that (A4) does not hold for $I_{4}$ in Figure 4.

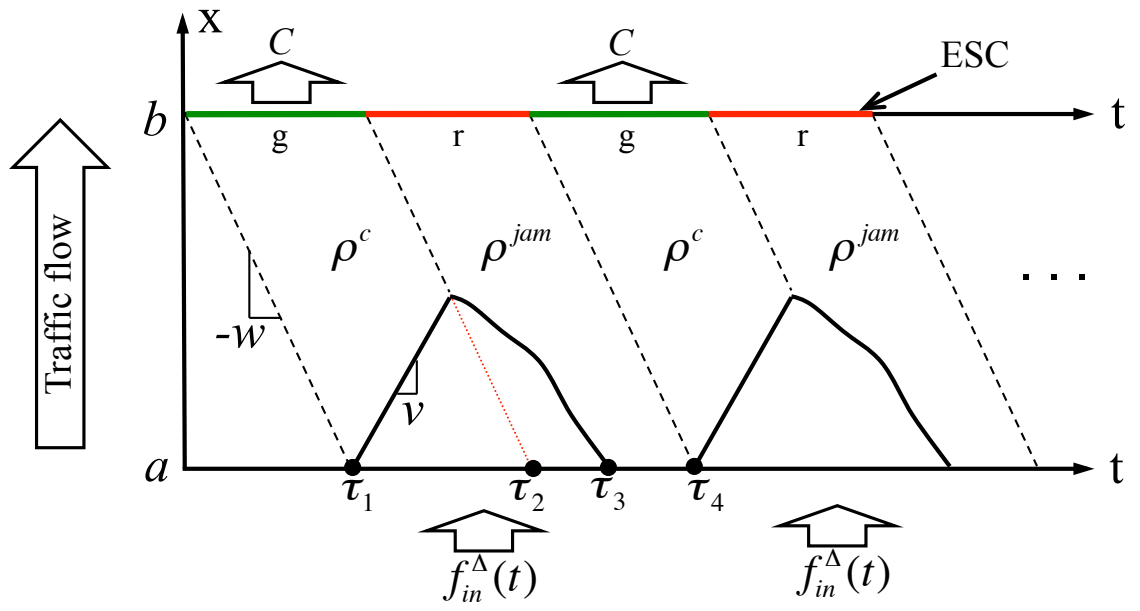

Figure 5: The space-time diagram of the link of interest, which triggers spillback. Notice that the control at the downstream end $x=b$ indicates the effective signal control $\mathbf{u}(t)$.

Under this assumption the density profile on the subject link is illustrated in Figure 5. According to (A4), the effective signal control (ESC) $\mathbf{u}(t)=1$ implies that the discharge flow is $C$. Thus the ESC creates two density values: 
ARTICLE LINK: http://www.sciencedirect.com/science/article/pii/S019126151500048X

PLEASE CITE THIS ARTICLE AS

Han, K., Gayah, V.V., 2015. Continuum signalized junction model for dynamic traffic networks: Offset, spillback, and multiple signal phases. Transportation Research Part B 77, 213-239.

$\rho^{c}$ (green phase) and $\rho^{\text {jam }}$ (red phase). Both densities propagate at a wave speed of $-w$. At time $\tau_{1}$, if $f_{\text {in }}^{\Delta}\left(\tau_{1}\right)<C$, then a shock wave will emerge and propagates forward into the link, at a constant speed $v$. After the shock intersects with the wave signaling the jam density, it propagates backward with possibly varying speed, depending on $f_{\text {in }}^{\Delta}(\cdot)$. The shock returns to the upstream boundary at $\tau_{3} \leq \tau_{4}$. ${ }^{4}$

Figure 5 suggests that, regardless of $f_{\text {in }}^{\Delta}(\cdot)$, there holds

$$
S(t)= \begin{cases}C & t \in\left[\tau_{1}, \tau_{3}\right] \\ 0 & t \in\left(\tau_{3}, \tau_{4}\right]\end{cases}
$$

We note that (4.23) holds true even when spillback is sustained, in which case the shock never leaves the upstream boundary $x=a$ and $\tau_{3}=\tau_{2}$.

Consequently, it is crucial to estimate $\tau_{3}$, which theoretically can take any value between $\tau_{2}$ and $\tau_{4}$. Due to the lack of further information, i.e. without a priori knowledge about $f_{\text {in }}^{\Delta}(\cdot)$, we will approximate $\tau_{3}$ with $\tilde{\tau}_{3} \doteq\left(\tau_{4}+\tau_{2}\right) / 2$, the middle point of $\tau_{2}$ and $\tau_{4}$. Such an approximation reduces the maximum potential error by half. Applying this idea to the most general case where one cycle of $\mathbf{u}(\cdot)$ may contain several green and red phases (see, for example, Section 4.1), we devise the following procedure for approximating $S(\cdot)$ based on assumption (A4).

\section{Algorithm I: Approximating $S(\cdot)$ given $u(\cdot)$ and $\mathcal{E}(\cdot)$}

Input Link length $L$; backward wave speed $w$; real-world signal control $u(\cdot)$; effective supply $\mathcal{E}(\cdot)$; and $\Omega$ defined in (4.19).

Step 1 Define the ESC $\mathbf{u}(t)=u(t) \cdot \mathcal{E}(t) / C$ with one cycle $\left[t_{0}, t_{0}+\Omega\right]$ (starting with a green phase) expressed as the union of green and red phases:

$$
\left[t_{0}, t_{0}+\Omega\right]=\bigcup_{j=1}^{m}\left[a_{j}, b_{j}\right] \cup \bigcup_{k=1}^{n}\left[c_{k}, d_{k}\right], \quad m, n \geq 1
$$

where the intervals $\left[a_{j}, b_{j}\right]$ and $\left[c_{k}, d_{k}\right]$ represent respectively the green and red phases of the ESC, $1 \leq$ $j \leq m, 1 \leq k \leq n$.

Step 2 Define and return the approximate supply function for $t \in\left[t_{0}+\frac{L}{w}, t_{0}+\Omega+\frac{L}{w}\right]$

$$
\tilde{S}(t) \doteq \begin{cases}C & t-\frac{L}{w} \in \bigcup_{j=1}^{m}\left[a_{j}, b_{j}\right] \cup \bigcup_{k=1}^{n}\left[c_{k}, \frac{d_{k}+c_{k}}{2}\right] \\ 0 & t-\frac{L}{w} \in \bigcup_{k=1}^{n}\left(\frac{d_{k}+c_{k}}{2}, d_{k}\right]\end{cases}
$$

We have the following proposition, which serves as the prerequisite of the GCSM as it verifies assumption (A3).

Proposition 4.9. Under assumption (A4), applying Algorithm I to all the links in the network guarantees that (A3) holds throughout the network. As a consequence, the GCSM can be applied in conjunction with Algorithm I.

Proof. If no spillback is present then (A3) is automatically true. In the case of spillback, the link that initiates it has a constant effective supply, which satisfies (A3). According to Algorithm I, the supply of this link $\tilde{S}(\cdot)$, defined as (4.24), is piecewise constant and periodic. Thus, the effective supplies of its upstream links satisfy (A3). A straightforward induction concludes this proposition.

\footnotetext{
${ }^{4}$ Otherwise, if $\tau_{3}>\tau_{4}$ then the supply function $S(\cdot)$ remains constant $C$ throughout a full cycle, and the problem reduces to the no-spillback case.
} 
ARTICLE LINK: http://www.sciencedirect.com/science/article/pii/S019126151500048X

PLEASE CITE THIS ARTICLE AS

Han, K., Gayah, V.V., 2015. Continuum signalized junction model for dynamic traffic networks: Offset, spillback, and multiple signal phases. Transportation Research Part B 77, 213-239.

\subsubsection{Method II for estimating supply $S(\cdot)$ given $u(\cdot)$ and $\mathcal{E}(\cdot)$}

The second method estimates the supply of the subject link by taking into account its interaction with upstream links in a more explicit way than Method I. We let $\mathcal{U}$ be the set of links upstream to the subject link. The following is assumed by the second method.

(A5). For any $I_{i} \in \mathcal{U}$, the discharge flow $f_{\text {out }, i}^{\Delta}(t)$ depends only on $u_{i}(t) \cdot \mathcal{E}_{i}(t)$ of that link.

Remark 4.10. Either one of the following conditions is sufficient for (A5) to hold.

(1) $D_{i}(t) \geq \mathcal{E}_{i}(t)$ for all $t$, so that $f_{\text {out }, i}^{\Delta}(t)=\min \left\{D_{i}(t), u_{i}(t) \cdot \mathcal{E}_{i}(t)\right\} \equiv u_{i}(t) \cdot \mathcal{E}_{i}(t)$.

(2) The exit of the upstream link $I_{i}$ remains congested (i.e. $D_{i}(t) \equiv C_{i}$ ) during its discharge window, so that $f_{\text {out }, i}^{\Delta}(t) \equiv \min \left\{C_{i}, u_{i}(t) \cdot \mathcal{E}_{i}(t)\right\}$.

Notably, the sustained spillback is also subsumed by (A5). For instance, in Figure 4 we have that for $i=4,7$,

$$
f_{\text {out }, i}^{\Delta}(t)= \begin{cases}C_{5} & \text { if } u_{i}(t) \cdot \mathcal{E}_{i}(t)>0 \\ 0 & \text { otherwise }\end{cases}
$$

Under assumption (A5), Algorithm II below computes the exact $S(\cdot)$.

\section{Algorithm II: Computing $S(\cdot)$ given $u(\cdot)$ and $\mathcal{E}(\cdot)$}

Input Link length $L$; backward wave speed $w$; real-world signal control $u(\cdot)$; effective supply $\mathcal{E}(\cdot)$; and $\Omega$ defined in (4.19). Let $\Delta_{u p}$ be the cycle length of the real-world signal at the upstream node; that is, $\Delta_{i}=\Delta_{u p}$, $\forall I_{i} \in \mathcal{U}$. Let $t_{0}$ be the beginning of a full cycle of $u(\cdot)$ (starting with the green phase) for the subject link.

Step 1 For $t \in\left[t_{0}+\frac{L}{w}, t_{0}+\frac{L}{w}+\operatorname{LCM}\left(\Omega, \Delta_{u p}\right)\right]$, solve the following algebraic equation system concerning the subject link.

if $\int_{t_{0}+\frac{L}{w}}^{t} f_{i n}^{\Delta}(\tau) d \tau=\int_{t_{0}}^{t-\frac{L}{w}} u(\tau) \cdot \mathcal{E}(\tau) d \tau$

then $S(t)=u\left(t-\frac{L}{w}\right) \cdot \mathcal{E}\left(t-\frac{L}{w}\right)$. Calculate $\mathcal{E}_{i}(t)$ for each upstream link $I_{i} \in \mathcal{U}$ and, in view of (A5), determine their discharge flows $f_{\text {out }, i}^{\Delta}(t)$ and hence the inflow $f_{\text {in }}^{\Delta}(t)=\sum_{I_{i} \in \mathcal{U}} f_{\text {out }, i}^{\Delta}(t)$ into the subject link.

else if $\int_{t_{0}+\frac{L}{w}}^{t} f_{i n}^{\Delta}(\tau) d \tau<\int_{t_{0}}^{t-\frac{L}{w}} u(\tau) \cdot \mathcal{E}(\tau) d \tau$

then $S(t)^{w}=C$. Calculate $\mathcal{E}_{i}(t)$ for each upstream link $I_{i} \in \mathcal{U}$ and, in view of (A5), determine their discharge flows and hence the inflow $f_{\text {in }}^{\Delta}(t)$.

end if

Step 2 Return $S(t)$ for $t \in\left[t_{0}+\frac{L}{w}, t_{0}+\frac{L}{w}+\operatorname{LCM}\left(\Omega, \Delta_{u p}\right)\right]$.

Unlike Algorithm I which only approximates the supply function, Algorithm II computes the exact $S(\cdot)$ using the variational principle. The reader is referred to Appendix $\mathrm{C}$ for a mathematical derivation of the algebraic equation system described in Algorithm II. This system can be easily solved with time-discretization; see Han et al. (2014) and Yerman et al. (2005) for some examples. Notice that this algebraic system only needs to be solved for one time period of $\operatorname{LCM}\left(\Omega, \Delta_{u p}\right)$, and the rest of $S(\cdot)$ is known from periodicity. Moreover, Algorithm II needs to be performed only once for a given set of signal timing parameters, and does not need to be repeated if the network is simulated multiple times using the same signal timing. The efficient implementation of Algorithm II in network simulation and optimization tasks will be demonstrated later in Section 6.

Similar to Method I, we have the following result concerning the validity of assumption (A3) in order for the GCSM to be applied. 
ARTICLE LINK: http://www.sciencedirect.com/science/article/pii/S019126151500048X

PLEASE CITE THIS ARTICLE AS

Han, K., Gayah, V.V., 2015. Continuum signalized junction model for dynamic traffic networks: Offset, spillback, and multiple signal phases. Transportation Research Part B 77, 213-239.

Proposition 4.11. Under assumption (A5), applying Algorithm II to all the links in the network guarantees that (A3) holds throughout the network. As a consequence, the GCSM can be applied in conjunction with Algorithm II.

Proof. If no spillback is present then (A3) is automatically true. In the presence of spillback, the link that initiates it has a constant effective supply $\mathcal{E}(\cdot)$, which satisfies (A3). According to (A5) and Algorithm II, its supply function $S(\cdot)$ is determined entirely by exogenous link/signal parameters and the periodic functions $\mathcal{E}(\cdot)$ and $u(\cdot)$. Thus, the resulting $S(\cdot)$ is also periodic. In addition, $S(\cdot)$ is obviously piecewise constant. We therefore conclude that the effective supplies of its upstream links satisfy (A3). Finally, the desired result follows from induction.

\subsection{Application of the GCSM and error estimates}

So far in this section, we have proposed a generalized continuum signal model (GCSM) for an arbitrary link given the knowledge of its effective supply $\mathcal{E}(\cdot)$ that satisfies (A3). Following this, we have presented two different methods for computing a link's supply $S(\cdot)$ and thus the effective supplies of its upstream links. Combining these two steps yields two continuum signal models that are general enough to not only handle both no-spillback and spillback cases, but also treat networks with a wide range of size and topology. In this section, we will demonstrate the use of the GCSM and analyze its approximation error on a given network, while noting that the techniques and insights are generalizable to any network size and topology.

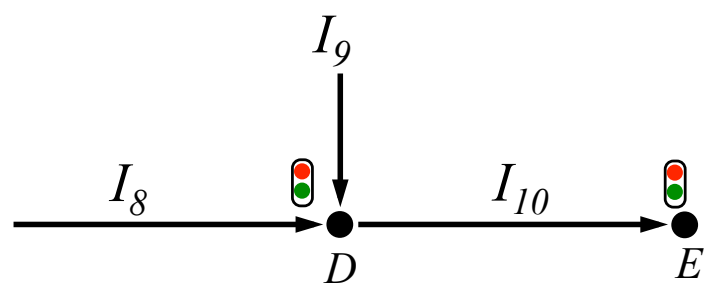

Figure 6: Signalized network with potential spillback at node $D$ triggered by $I_{10}$.

Without loss of generality, we focus on the network depicted in Figure 6, where link $I_{10}$ may trigger spillback at node $D$. We will mainly present and analyze the continuum signal model for $I_{8}$, while noting that link $I_{9}$ can be treated in a symmetric and similar way. Moreover, link $I_{10}$ can be treated with the GCSM assuming the absence of spillback from downstream, as node $E$ is represented as the "sink" of the network. We begin by articulating the continuum model for $I_{8}$ in the presence of spillback.

Example 1. (GCSM+Algorithm I) The continuum signal model for $I_{8}$ is devised as follows. As (A4) automatically holds for $I_{10}$, we first apply Algorithm I (Section 4.3.1) to compute the approximate supply $\tilde{S}_{10}(\cdot)$ with inputs $u_{10}(\cdot)$ and $\mathcal{E}_{10}(\cdot) \equiv C_{10}$. Then, we compute the effective supply $\tilde{\mathcal{E}}_{8}(t) \doteq \min \left\{C_{8}, \tilde{S}_{10}(t)\right\}$. Finally, we apply the GCSM to $I_{8}$ with inputs $\tilde{\mathcal{E}}_{8}(\cdot)$ and $u_{8}(\cdot)$.

Example 2. (GCSM+Algorithm II) Assuming (A5) for link $I_{10}$, the continuum approximation for $I_{8}$ is devised as follows. We first apply Algorithm II (Section 4.3.2) to compute $S_{10}(\cdot)$ with inputs $u_{10}(\cdot)$ and $\mathcal{E}_{10}(\cdot) \equiv C_{10}$. Then, we compute the effective supply $\mathcal{E}_{8}(t) \doteq \min \left\{C_{8}, S_{10}(t)\right\}$. Finally, we apply the GCSM to $I_{8}$ with inputs $\mathcal{E}_{8}(\cdot)$ and $u_{8}(\cdot)$.

A more concrete numerical demonstration of these two examples will be given in Section 6.1.1. The next two theorems perform the error estimation for these two continuum signal models.

Theorem 4.12. (Error estimate for GCSM+Algorithm I) Consider the continuum approximation for $I_{8}$ described in Example 1. Then the resulting Moskowitz functions satisfy:

$$
\left|N_{8}^{0}(t, x)-N_{8}^{\Delta}(t, x)\right| \leq \zeta_{8}\left(1-\zeta_{8}\right) L C M\left(\Delta_{8}, \Delta_{10}\right) \min \left\{C_{8}, C_{10}\right\}+\min \left\{\frac{1}{2}\left(1-\eta_{10}\right), \eta_{8}\right\} \min \left\{C_{8}, C_{10}\right\} t
$$

where $N_{8}^{0}(t, x)$ is the Moskowitz function corresponding to GCSM+Algorithm I. $\zeta_{8}$ is the green split of the ESC $\mathbf{u}_{8}(\cdot)$ determined by $u_{8}(\cdot)$ and $\tilde{\mathcal{E}}_{8}(\cdot) . \eta_{8}$ and $\eta_{10}$ are the green splits of the real-world signals $u_{8}(\cdot)$ and $u_{10}(\cdot)$, respectively. 
ARTICLE LINK: http://www.sciencedirect.com/science/article/pii/S019126151500048X

PLEASE CITE THIS ARTICLE AS

Han, K., Gayah, V.V., 2015. Continuum signalized junction model for dynamic traffic networks: Offset, spillback, and multiple signal phases. Transportation Research Part B 77, 213-239.

Proof. We refer the reader to Appendix B.3 for the proof.

Theorem 4.13. (Error estimate for GCSM+Algorithm II) Consider the continuum approximation for $I_{8}$ described in Example 2. Then the Moskowitz functions satisfy:

$$
\left|N_{8}^{0}(t, x)-N_{8}^{\Delta}(t, x)\right| \leq \zeta_{8}\left(1-\zeta_{8}\right) L C M\left(\Delta_{8}, \Delta_{10}\right) \min \left\{C_{8}, C_{10}\right\}
$$

where $N_{8}^{0}(t, x)$ is the Moskowitz function corresponding to GCSM+Algorithm II. $\zeta_{8}$ is the green split of the ESC $\mathbf{u}_{8}(\cdot)$ determined by $u_{8}(\cdot)$ and $\mathcal{E}_{8}(\cdot)$.

Proof. Under the assumption (A5), the supply function estimated from Algorithm II coincides with the exact supply $S_{10}(\cdot)$. Repeating the proof of Theorem 4.12 with $S_{10}(t) \equiv \tilde{S}_{10}(t)$ yields the desired result.

Remark 4.14. The two models defined in Example 1 and 2 as well as their respective error estimates in Theorem 4.12 and 4.13 apply to sustained and transient spillbacks alike. The error bound (4.25) for the first model (GCSM+Method I) contains a constant and a part that grows with time; the latter is caused by the inexactness of the supply estimation performed by Algorithm I. The error bound (4.26) for the second model (GCSM+Method II), on the other hand, is uniformly bounded and independent of time. Recall the error bound for the Han et al. (2014) model:

$$
\eta_{8}\left(1-\eta_{8}\right) \Delta_{8} \min \left\{C_{8}, C_{10}\right\}+\min \left\{C_{8}, C_{10}\right\} \eta_{8} t \quad \text { (Theorem } 5.4 \text { of Han et al. (2014)) }
$$

A comparison of its time-varying part with the time-varying part of (4.25) suggests that the error of GCSM + Algorithm I is at most as large as the Han et al. (2014) model, and its error is strictly smaller if $\frac{1}{2}\left(1-\eta_{10}\right)<\eta_{8}$. On the other hand, GCSM+Algorithm II represents a significant improvement over the Han et al. (2014) model in terms of approximation accuracy as it yields uniformly bounded error for both sustained and transient spillback cases. All these models will be rigorously tested using a battery of simulations later in Section 6.1.2.

We next describe in further detail the procedure for simulating traffic on the network shown in Figure 6 using the newly proposed models. This will be in the form of a pseudo code, and presented in Algorithm III.

\section{Algorithm III: Continuum signal model for the network in Figure 6.}

Input Link parameters; signal controls $u_{8}(t), u_{9}(t)=1-u_{8}(t)$, and $u_{10}(t)$, which include cycle lengths, green splits, and offsets.

Step 1 Apply Algorithm I or II to compute the supply $S_{10}(\cdot)$ of $I_{10}$ in the presence of spillback, with input $u_{10}(\cdot)$ and $\mathcal{E}_{10}(\cdot) \equiv C_{10}$.

Step 2 For $i=8,9$, calculate the effective supplies $\mathcal{E}_{i}(t)=\min \left\{C_{i}, S_{10}(t)\right\}$. Then compute

$$
R_{i}=\frac{1}{\operatorname{LCM}\left(\Delta_{8}, \Delta_{10}\right)} \int_{t_{0}}^{t_{0}+L C M\left(\Delta_{8}, \Delta_{10}\right)} u_{i}(t) \cdot \mathcal{E}_{i}(t) d t
$$

where $t_{0}$ is arbitrary.

Step 3 Perform traffic simulation with the following rule:

if $N_{u p, 10}^{0}(t)<N_{\text {down }, 10}^{0}\left(t-\frac{L_{10}}{w_{10}}\right)+\rho_{10}^{\text {jam }} L_{10} \quad$ (no spillback)

then propagate traffic dynamics with the following boundary conditions

$$
f_{\text {out }, i}^{0}(t)=\min \left\{D_{i}(t), \eta_{i} \min \left\{C_{i}, C_{10}\right\}\right\} \quad i=8,9
$$

else if $N_{u p, 10}^{0}(t)=N_{d o w n, 10}^{0}\left(t-\frac{L_{10}}{w_{10}}\right)+\rho_{10}^{j a m} L_{10} \quad$ (spillback)

then propagate traffic dynamics with the following boundary conditions

$$
f_{\text {out }, i}^{0}(t)=\min \left\{D_{i}(t), R_{i}\right\} \quad i=8,9
$$

end if 


\section{ARTICLE LINK: http://www.sciencedirect.com/science/article/pii/S019126151500048X}

\section{PLEASE CITE THIS ARTICLE AS}

Han, K., Gayah, V.V., 2015. Continuum signalized junction model for dynamic traffic networks: Offset, spillback, and multiple signal phases. Transportation Research Part B 77, 213-239.

As a concluding remark for this section, we observe that the GCSM handles spillback by estimating the supply profiles of downstream links and analyzing their interactions with upstream signal controls and discharge flows. Within this process the GCSM employs signal offsets to accurately capture these complex interactions.

\section{Extension to realistic signalized intersections}

In this section, we qualitatively discuss how the continuum signal model can be extended to account for a variety of intersection configurations. These include multiple entry links into the intersection, multiple exit links from the intersection, turn-lanes, and complex phasing schemes. We consider here an isolated signalized intersection for simplicity, noting that the impacts of offsets and queue spillbacks from adjacent signals can be accommodated in a manner similar to what has been described in previous sections.

Multiple entry links: Multiple (more than one) entry links into an intersection can be modeled in a relatively straightforward fashion. In this case, the discharge window for each of these links would need to be calculated separately to model vehicle throughput on each. Care would be needed to account for the fact that each entry link would experience a different signal offset with respect to any adjacent signal.

Multiple exit links: Most of the illustrative examples provided thus far has considered vehicles discharging from the signalized intersection onto a single downstream link. However, multiple discharge links can be accommodated in the effective supply function for a particular approach link as in (2.3) if the fraction of vehicles using each of these downstream links are known a priori for each signalized intersection. Of course, this is not practical in many networks where origin-destination pairs are specified and vehicles seek to use the shortest route to complete their trip. In this, more realistic, case, dynamic network loading methods (e.g., Friesz et al., 2013) can be integrated to predict how vehicles choose between competing routes. More details will be provided on this latter approach in a future publication.

Turn-lanes: Dedicated turn-lanes should be modeled using multiple links upstream of the intersection that each represent individual lane movements. For example, Figure 7 shows a link-based representation that can be used to model a typical intersection approach that contains a single left-turn lane and two through lanes. Note that an unsignalized diverge would be needed where the left-turn lane begins to capture the impacts of queue spillbacks from either the left-turn or through lanes that might block the adjacent movement.
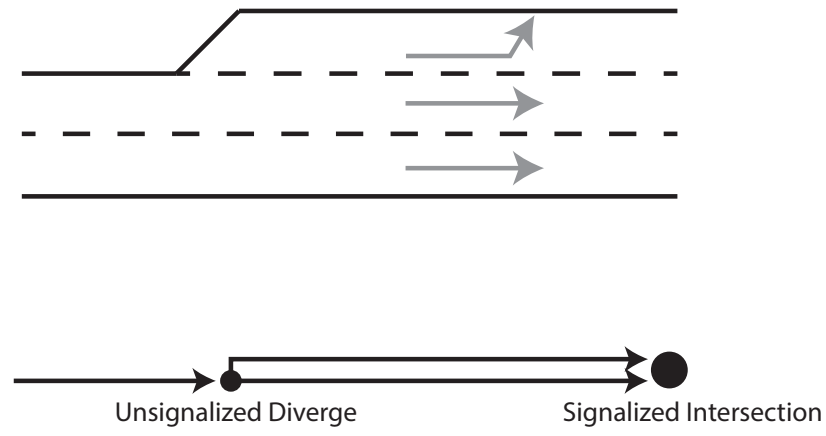

Figure 7: Example of turn lane treatment. Top: Schematic of intersection approach; bottom: Link representation that includes turn lane and unsignalized diverge junction.

Complex phasing schemes: Consider the complex phasing scheme for a particular approach shown in Figure 8 in which solid lines represent protected movements while dotted lines represent permitted movements. For this complex phase, two sub-phases are considered: $\phi_{1 A}$, which allows protected left-turn and through movements, and $\phi_{1 B}$, which allows protected through movement but permitted left-turns. Let us consider only the movements from the left-hand approach. The two movements possible (left-turn and through) can be separated and modeled independently. The through movement will be assumed to have a simple control function, $u_{T}(t)$, that permits movement during both subphases. The left-turn movement is more complicated. During the protected left-turn phase $\left(\phi_{1 A}\right)$, the control function, $u_{L}(t)$, will be treated as before. However, during the permitted phase $\left(\phi_{1 B}\right)$, the capacity at the intersection allocated to the left-turn movement would need to be adjusted to account for the reduced discharge rate experienced due to 
ARTICLE LINK: http://www.sciencedirect.com/science/article/pii/S019126151500048X

PLEASE CITE THIS ARTICLE AS

Han, K., Gayah, V.V., 2015. Continuum signalized junction model for dynamic traffic networks: Offset, spillback, and multiple signal phases. Transportation Research Part B 77, 213-239.

opposing traffic. This could be a fixed value assumed to be known a priori (less realistic) or calculated dynamically as a function of the opposing through volumes using a simple gap acceptance model (more realistic).
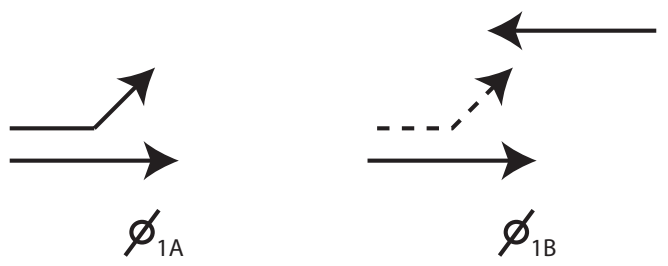

Figure 8: Example of complex intersection phasing scheme that can be accommodated.

Pedestrian and all-red phases: Many signals also have all-red periods that are implemented for safety and/or pedestrian movements across all approaches. This can be easily incorporated into the continuum model as a fraction of the cycle in which no vehicles are allowed to discharge through the intersection. With this extension, the sum of the $\eta_{i}$ values for each individual approach would be less than one to reflect the fact that the entire cycle cannot be used for vehicle discharge.

\subsection{Test on a realistic signalized junction}

We will test the continuum signal model on a realistic intersection shown in Figure 9(a). Figure 9(b) shows the signal stages and corresponding vehicle movements. Notice that a pedestrian stage is included. The signal timing plan is summarized in Table 1 .

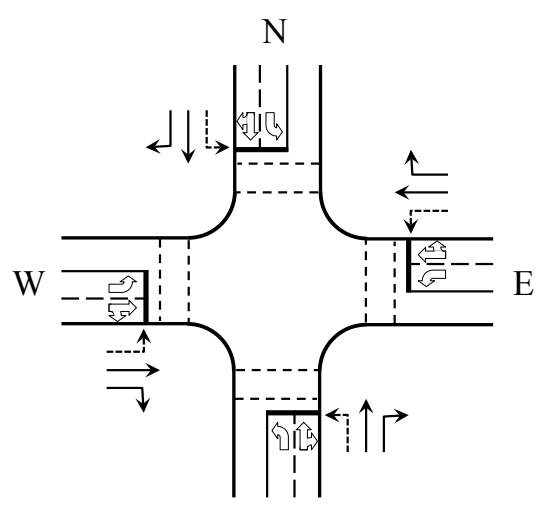

$\mathrm{S}$

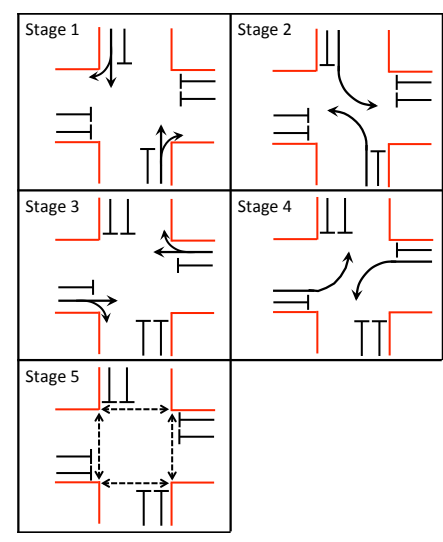

(b)

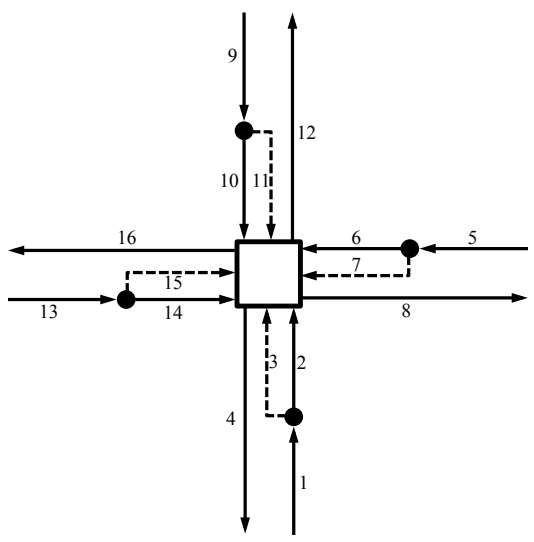

(c)

Figure 9: A realistic junction geometry (a) and signal stage plan (b). A simplified graph representation of this junction is shown in (c), where the dashed arrows represent left-turn lanes and the solid dots represent unsignalized diverge nodes.

\begin{tabular}{|c|c|c|c|c|c|c|}
\hline & Stage 1 & Stage 2 & Stage 3 & Stage 4 & Stage 5 & Total per cycle \\
\hline All-red (s) & 4 & 4 & 4 & 4 & 4 & 20 \\
\hline Green (s) & 26 & 11 & 37 & 11 & 15 & 100 \\
\hline Duration (s) & 30 & 15 & 41 & 15 & 19 & 120 \\
\hline
\end{tabular}

Table 1: Signal timing plan for the junction shown in Figure 9.

According to our discussion above, the left-turn lanes can be modeled by additional upstream links and unsignalized diverge nodes, which are respectively shown as dashed arrows and solid dots in Figure 9(c). Let us use the 


\section{ARTICLE LINK: http://www.sciencedirect.com/science/article/pii/S019126151500048X}

\section{PLEASE CITE THIS ARTICLE AS}

Han, K., Gayah, V.V., 2015. Continuum signalized junction model for dynamic traffic networks: Offset, spillback, and multiple signal phases. Transportation Research Part B 77, 213-239.

southern approach as an example to demonstrate the continuum signal model. The green time for the through movement and the right turn (Stage 1) is $26 \mathrm{~s}$ in a full cycle. Thus, in the continuum signal model the exit flow of link 2 (see Figure 9(c)) can be expressed as

$$
f_{\text {out }, 2}^{0}(t)=\min \left\{D_{2}(t), \frac{26}{120} \cdot \mathcal{E}_{2}(t)\right\}=\min \left\{D_{2}(t), \frac{26}{120} \cdot \min \left\{C_{2}, \frac{C_{8}}{\alpha_{2,8}}, \frac{C_{12}}{\alpha_{2,12}}\right\}\right\}
$$

where $\alpha_{2,8}$ and $\alpha_{2,12}$ are the vehicle turning percentages. For link 3, the exit flow is expressed as (notice that cars are discharged from link 3 in stage 2)

$$
f_{\text {out }, 3}^{0}(t)=\min \left\{D_{3}(t), \frac{11}{120} \cdot \mathcal{E}_{3}(t)\right\}=\min \left\{D_{3}(t), \frac{11}{120} \cdot \min \left\{C_{3}, C_{16}\right\}\right\}
$$

The continuum model for the other arms of the junction is similarly defined. Notice that (5.28)-(5.29) are for the nospillback case; the impacts of queue spillbacks and offsets from adjacent signals can be accommodated in a manner similar to what has been described in previous sections.

The continuum signal model has a significant advantage over the on-and-off signal model in terms of computational efficiency. In particular, given the signal timing in Table 1, the time step chosen for the on-and-off model must not exceed $1 \mathrm{~s}$. On the other hand, the continuum model can accommodate much larger time steps, which are not constrained by the specific signal timing plan. We use data collected at a real-world junction, ${ }^{5}$ including inflows and time-varying turning percentages, and simulate traffic for a period of two hours in the morning peak (7:30-9:30 am). The time steps employed are $1 \mathrm{~s}$ for the on-and-off model and $10 \mathrm{~s}$ for the continuum model. The resulting computational times are $19 \mathrm{~s}$ (on-and-off) and $0.31 \mathrm{~s}$ (continuum), which shows the superior computational efficiency of the continuum model for simulating realistic signal controls.

Figure 10 compares the on-and-off model with the continuum model in terms of cumulative vehicles entering the intersection (left) and exiting the intersection (right). It can be seen that the continuum model captures the aggregate throughput of the intersection and shows remarkable consistency with the on-and-off model despite its much larger time step. Furthermore, the continuum approximation error is uniformly bounded and does not grow with time, as predicted by our theory.
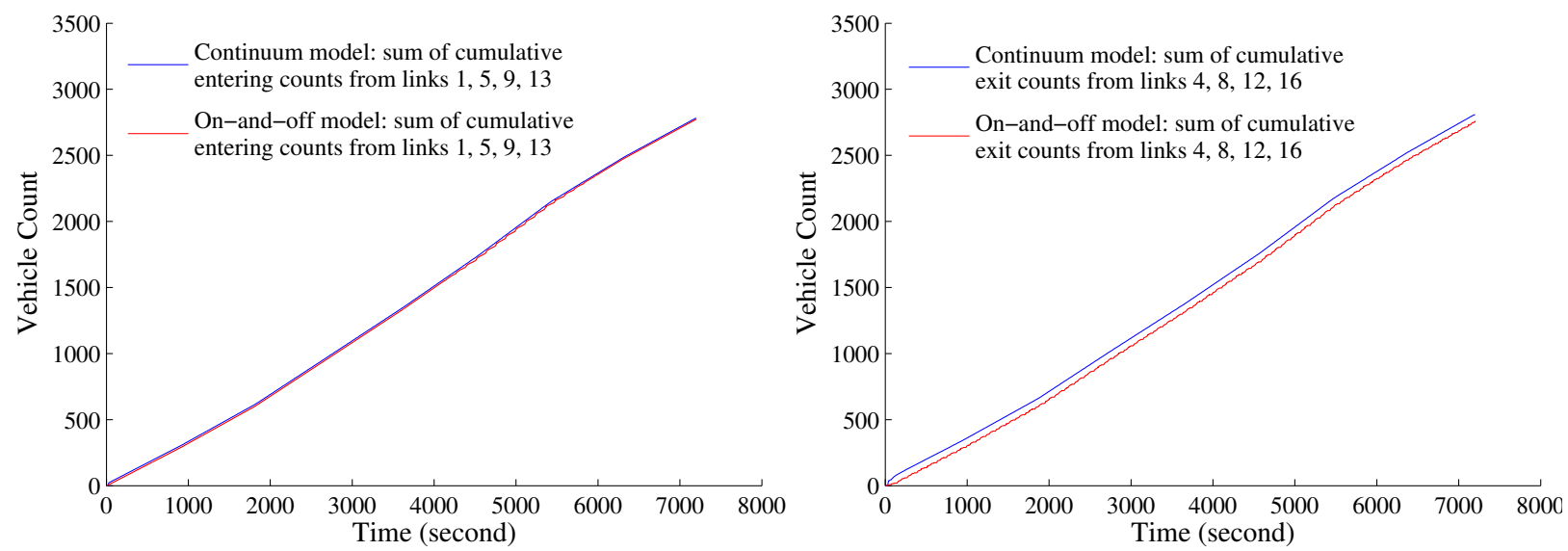

Figure 10: Left: sum of cumulative entering vehicle counts on links 1, 5, 9 and 13 (into the intersection). Right: sum of cumulative exiting vehicle counts of links 4, 8, 12 and 16 (out of the intersection).

\footnotetext{
${ }^{5}$ The traffic data are collected at a junction in the west end of Glasgow, Scotland, by Sky High Count On Us http://skyhightechnology.com/. These data have been converted to be consistent with the right-hand driving road intersection shown in Figure 9.
} 


\section{ARTICLE LINK: http://www.sciencedirect.com/science/article/pii/S019126151500048X}

\section{PLEASE CITE THIS ARTICLE AS}

Han, K., Gayah, V.V., 2015. Continuum signalized junction model for dynamic traffic networks: Offset, spillback, and multiple signal phases. Transportation Research Part B 77, 213-239.

\section{Numerical examples of the GCSM}

This section consists of two parts. The first part (Section 6.1) presents several numerical studies of the generalized continuum signal model (GCSM) and accompanying methods (Algorithm I \& II), for simulating the aggregate network flows in the presence of vehicle spillback. The second part (Section 6.2) describes a signal optimization procedure based on metaheuristics, which employs the traditional on-and-off model and the continuum model proposed here. It will be shown that the continuum model is not only readily applicable for solving signal optimization problems, but is also computationally more efficient than the on-and-off model and yields improved solution quality at the same time.

In our numerical calculations, the network dynamics employ the link transmission model (Yerman et al., 2005). All computations were performed on a standard laptop with 4GM RAM. ${ }^{6}$

\subsection{The GCSM for handling spillback}

\subsubsection{Simple network}

We provide a numerical demonstration of the GCSM and Algorithm I and II based on the same network shown in Figure 6. In particular, we will carry out Algorithm III in detail, while showing relevant quantities along the procedure. The link and signal parameters are summarized in Table 2 , where it is agreed that $u_{9}(t)=1-u_{8}(t)$ and thus parameters of $u_{9}(\cdot)$ are not shown.

\begin{tabular}{|c|c|c|c|c|c|c||c|c|c|c|}
\hline Link & $v_{i}$ & $w_{i}$ & $\rho_{i}^{c}$ & $\rho_{i}^{j a m}$ & $C_{i}$ & $L_{i}$ & Signal & $\Delta_{i}$ & $\eta_{i}$ & Offset \\
\hline$I_{8}, I_{9}$ & $40 / 3$ & $40 / 9$ & 0.075 & 0.3 & 1 & 200 & $u_{8}(\cdot)$ & 60 & $1 / 3$ & $O_{8,10}=35$ \\
\hline$I_{10}$ & $40 / 3$ & $40 / 9$ & 0.1 & 0.4 & $4 / 3$ & 200 & $u_{10}(\cdot)$ & 120 & $1 / 2$ & - \\
\hline
\end{tabular}

Table 2: Link and signal control parameters for the network shown in Figure 6. $v_{i}$ and $w_{i}$ in meter/s, $\rho_{i}^{c}$ and $\rho_{i}^{j a m}$ in veh/meter, $C_{i}$ in veh/s, $L_{i}$ in meter, $\Delta_{i}$ and $O_{8,10}$ in s.

In order to apply the GCSM to handle spillback, we first need to calculate the supply function of the link that triggers spillback, i.e. $I_{10}$. This is done using Algorithms I and II by following the procedures described in Example 1 and 2 respectively; see Section 4.4. And the resulting $S_{10}(\cdot)$ are calculated as:

$$
\begin{aligned}
& \tilde{S}_{10}(t)=\left\{\begin{array}{ll}
C_{10} & t \in\left[t_{10}+\frac{L_{10}}{w_{10}}, t_{10}+\frac{L_{10}}{w_{10}}+90\right] \\
0 & t \in\left[t_{10}+\frac{L_{10}}{w_{10}}+90, t_{10}+\frac{L_{10}}{w_{10}}+120\right]
\end{array} \quad\right. \text { (Algorithm I) } \\
& S_{10}(t)=\left\{\begin{array}{ll}
C_{10} & t \in\left[t_{10}+\frac{L_{10}}{w_{10}}, t_{10}+\frac{L_{10}}{w_{10}}+80\right] \\
0 & t \in\left[t_{10}+\frac{L_{10}}{w_{10}}+80, t_{10}+\frac{L_{10}}{w_{10}}+120\right]
\end{array} \quad\right. \text { (Algorithm II) }
\end{aligned}
$$

where $t_{10}$ is the start of any cycle (and green phase) of $u_{10}(\cdot) . \tilde{S}_{10}(\cdot)$ and $S_{10}(\cdot)$ are periodic with periods equal to $\operatorname{LCM}\left(\Delta_{8}, \Delta_{10}\right)=120 \mathrm{~s}$. From this we define the effective supplies $\tilde{\mathcal{E}}_{8}(t)=\min \left\{C_{8}, \tilde{S}_{10}(t)\right\}, \mathcal{E}_{8}(t)=\min \left\{C_{8}, S_{10}(t)\right\}$; the effective supplies for $I_{9}$ are similarly defined. Following the definition of the GCSM (Definition 4.5) using the additional knowledge of $u_{8}(\cdot)$ and $u_{9}(\cdot)$, which includes cycle, split and offset, we readily calculate that

$$
\begin{array}{lll}
f_{\text {out }, 8}^{0}(t)=\min \left\{D_{8}(t), \frac{20}{120} \min \left\{C_{8}, C_{10}\right\}\right\}, & f_{\text {out }, 9}^{0}(t)=\min \left\{D_{9}(t), \frac{70}{120} \min \left\{C_{9}, C_{10}\right\}\right\} & \text { (Alg. I) } \\
f_{\text {out }, 8}^{0}(t)=\min \left\{D_{8}(t), \frac{20}{120} \min \left\{C_{8}, C_{10}\right\}\right\}, & f_{\text {out }, 9}^{0}(t)=\min \left\{D_{9}(t), \frac{60}{120} \min \left\{C_{9}, C_{10}\right\}\right\} & \text { (Alg. II) }
\end{array}
$$

where in each fraction above, the numerator is the length of the discharge window in one cycle of the ESC, and the denominator is the cycle length of the ESC, which is equal to $\operatorname{LCM}\left(\Delta_{i}, \Delta_{10}\right), i=8$, 9. Figure 11 shows $N_{d o w n, 8}^{0}(t)$ and $N_{\text {down, } 9}^{0}(t)$ computed from (6.30) and (6.31). For comparison we also include the result obtained from the Han et al. (2014) model (2.5). It can be seen that the estimation errors of the proposed models are correctly bounded by the

\footnotetext{
${ }^{6}$ All tests were performed using Matlab. The original Matlab codes for all tests are available on the authors' respective websites for use by any interested reader.
} 


\section{ARTICLE LINK: http://www.sciencedirect.com/science/article/pii/S019126151500048X}

\section{PLEASE CITE THIS ARTICLE AS}

Han, K., Gayah, V.V., 2015. Continuum signalized junction model for dynamic traffic networks: Offset, spillback, and multiple signal phases. Transportation Research Part B 77, 213-239.

theoretical upper and lower bounds. In the case of GCSM+Algorithm I, the error grows with time, as does the error for the Han et al. (2014) model. In the case of GCSM+Algorithm II, the error is uniformly bounded and does not grow with time, indicating remarkable consistency between the on-and-off model and the proposed model, as guaranteed by the theory (Theorem 4.13). Furthermore, we notice that the green splits of the effective signal controls, shown as the fractions in (6.30) and (6.31), are very different from the splits of the real-world signal controls $(1 / 3$ and $2 / 3)$; thus models based solely on the real-world signal splits, such as (2.5), cannot capture well the aggregate traffic dynamics at signalized intersections impacted by spillback.
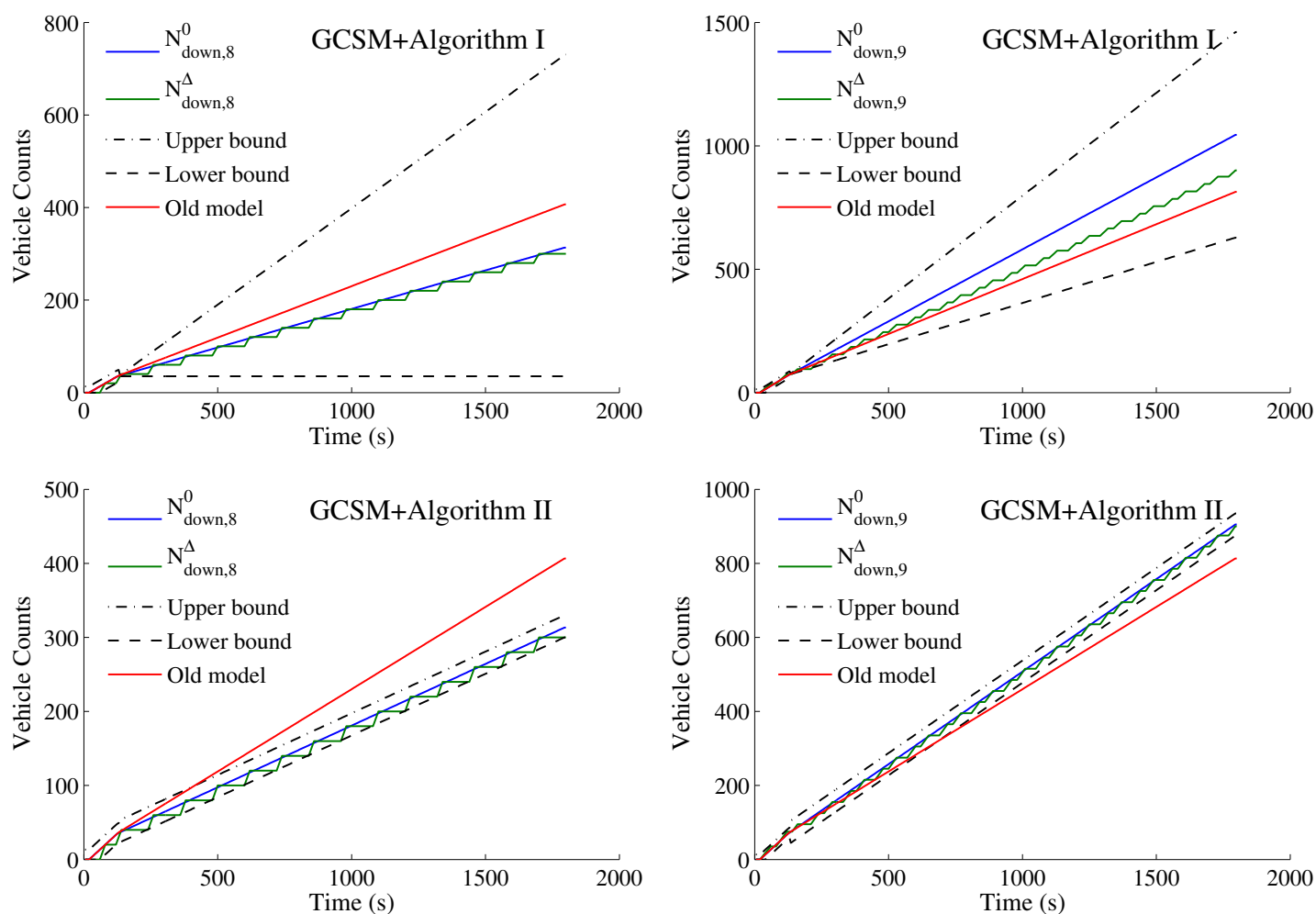

Figure 11: Comparison of GCSM+Algorithm I (top row) and GCSM+Algorithm II (bottom row), with the old continuum signal model (Han et al., 2014). Left column: cumulative exit vehicle counts on $I_{8}$; right column: cumulative exit vehicle counts on $I_{9}$.

The computation depicted in Figure 11 has a time horizon of $1800 \mathrm{~s}$ (30 min), and employs a $5 \mathrm{~s}$ time step for the on-and-off model and a $15 \mathrm{~s}$ time step for the continuum models. Notice that the former time step is restricted by the given signal timing to be implemented; and in this case should be no more than $5 \mathrm{~s}$ in view of Table 2 . The computational times of a complete simulation run are $0.035 \mathrm{~s}$ (on-and-off) and $0.011 \mathrm{~s}$ (continuum), where the latter is averaged over three continuum models considered here. The computational time is naturally related, if not entirely inversely proportional to, the time step size, as the number of numerical operations needed in the link transmission model is almost linear with respect to the number of time intervals. Thus, the flexibility of the continuum models in accommodating significantly larger time steps can substantially reduce the computational effort.

\subsubsection{Larger network}

We perform an extensive test on a larger network of the three continuum models: (2.5) proposed by Han et al. (2014), GCSM+Algorithm I, and GCSM+Algorithm II. The purpose is to statistically evaluate the approximation accuracy of these three models under various congestion levels and demand profiles. The network depicted in Figure 12 is considered. In order to set up GCSM+Algorithm I and GCSM+Algorithm II for this network, we consider the following four scenarios:

1. no spillback anywhere; 
ARTICLE LINK: http://www.sciencedirect.com/science/article/pii/S019126151500048X

PLEASE CITE THIS ARTICLE AS

Han, K., Gayah, V.V., 2015. Continuum signalized junction model for dynamic traffic networks: Offset, spillback, and multiple signal phases. Transportation Research Part B 77, 213-239.

2. spillback at node $\mathrm{G}$ only;

3. spillback at node F only;

4. spillback at both $\mathrm{F}$ and $\mathrm{G}$.

The continuum signal model needs to be calibrated for each scenario. And, the invocation of each scenario is done through the logical expressions similar to those in Algorithm III.

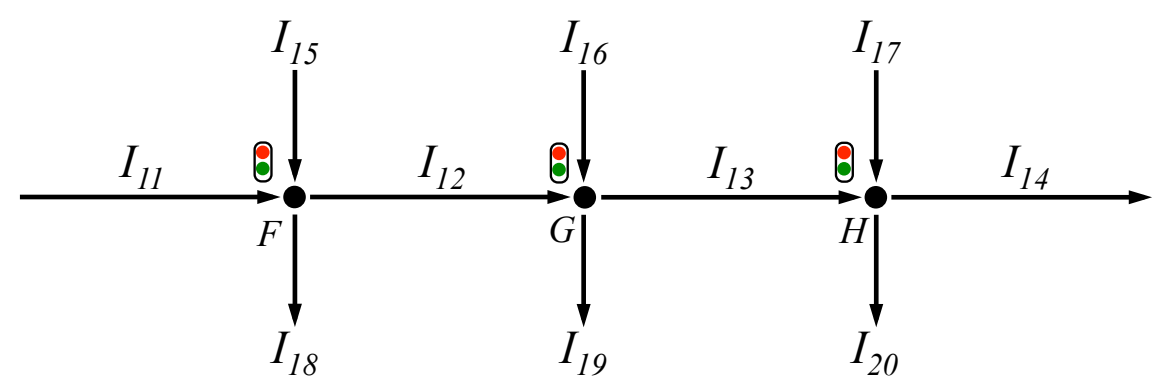

Figure 12: The test network with three signalized junctions and possible spillbacks at nodes $F$ and $G$.

All the links in Figure 12 are assumed to share the same parameters as link $I_{10}$ in Table 2 . In terms of the signal timing parameters, we assume that all signals $u_{11}(\cdot), u_{12}(\cdot)$ and $u_{13}(\cdot)$ have the same cycle length of $60 \mathrm{~s}$ and green split of $1 / 2$. Moreover, the offsets are: $O_{11,12}=25 \mathrm{~s}, O_{12,13}=25 \mathrm{~s}$. Fixed vehicle turning ratios are assumed: $\alpha_{11,12}=\alpha_{15,12}=\alpha_{12,13}=\alpha_{16,13}=\alpha_{13,14}=\alpha_{17,14}=0.6$. The time step employed by the on-and-off model is $5 \mathrm{~s}$, and the time step employed by all three continuum models is $15 \mathrm{~s}$.

Given this problem setup, we test the three continuum models using a battery of simulation runs. Each simulation has a time horizon of two hours, with network inflows on $I_{11}, I_{15}, I_{16}$ and $I_{17}$ randomly generated between zero and the flow capacity. As a performance measure for the accuracy of these continuum signal models, we consider

$$
\max _{i=11, \ldots, 20}\left\{\frac{\max _{t \in[0,7200], x \in\left[a_{i}, b_{i}\right]}\left|N_{i}^{\Delta}(t, x)-N_{i}^{0}(t, x)\right|}{N_{d o w n, i}^{\Delta}(7200)}\right\}
$$

which measures, among all links $I_{11}-I_{20}$, the maximum continuum approximation error on a single link relative to this link's total throughput in two hours: $N_{d o w n, i}^{\Delta}(7200)$. Each model is tested with 1000 simulation runs, and the mean and standard deviation of the performance measure (6.32) is summarized in Table 3.

\begin{tabular}{|c|c|c|c|}
\hline & Mean & Standard deviation & Average CPU time \\
\hline Han et al. (2014) model & $24.26 \%$ & $13.68 \%$ & $0.075 \mathrm{~s}$ \\
\hline GCSM+Algorithm I & $11.34 \%$ & $4.94 \%$ & $0.075 \mathrm{~s}$ \\
\hline GCSM+Algorithm II & $4.39 \%$ & $1.18 \%$ & $0.075 \mathrm{~s}$ \\
\hline
\end{tabular}

Table 3: Summary of the performance measure (6.32) with 1000 simulation runs. The CPU time is based on a time step of $15 \mathrm{~s}$ for all three continuum models. For the same simulation task, the on-and-off model takes $0.43 \mathrm{~s}$ on average, based on a time step of $5 \mathrm{~s}$.

We conclude from these simulation results that the two continuum signal models proposed in this paper can properly handle complex spillback occurrences at a network with significantly improved approximation accuracy than the Han et al. (2014) model. In particular, the GCSM+Algorithm II model has the best performance and reduces the error of the Han et al. (2014) model by $82 \%$; this is followed by the GCSM+Algorithm I model, which has an error reduction of $51 \%$. These errors are further broken down by link; that is, we consider

$$
\frac{\max _{t \in[0,7200], x \in\left[a_{i}, b_{i}\right]}\left|N_{i}^{\Delta}(t, x)-N_{i}^{0}(t, x)\right|}{N_{\text {down,i }}^{\Delta}(7200)}, \quad i=11, \ldots, 20
$$




\section{ARTICLE LINK: http://www.sciencedirect.com/science/article/pii/S019126151500048X}

\section{PLEASE CITE THIS ARTICLE AS}

Han, K., Gayah, V.V., 2015. Continuum signalized junction model for dynamic traffic networks: Offset, spillback, and multiple signal phases. Transportation Research Part B 77, 213-239.

These are averaged for each link and shown in Table 4. It can be seen that the worst-case errors occur on link $I_{11}$, whose continuum approximation is mostly affected by spillback that originates from $I_{13}$ and propagates through two junctions $G$ and $F$. The reason is that the approximation error for the spillback case may add up from one link to the other in the direction of spillback. Indeed, the closer a link is to the origin of the spillback, the smaller its approximation error is. From the table we also see that for most links in the network the continuum approximation errors are well below the worst-case errors as appearing in Table 3.

\begin{tabular}{|c|c|c|c|c|c|c|}
\hline & $I_{11}(\%)$ & $I_{12}(\%)$ & $I_{13}(\%)$ & $I_{15}(\%)$ & $I_{16}(\%)$ & $I_{17}(\%)$ \\
\hline Han et al. (2014) model & 24.26 & 8.09 & 2.33 & 7.20 & 5.59 & 0.45 \\
\hline GCSM+Algorithm I & 11.32 & 5.69 & 1.87 & 4.05 & 3.61 & 0.46 \\
\hline GCSM+Algorithm II & 4.24 & 2.14 & 1.15 & 2.35 & 0.5 & 0.42 \\
\hline
\end{tabular}

Table 4: Summary of the performance measure (6.33) for each link with 1000 simulation runs

Figure 13 shows, for one particular simulation run of the GCSM+Algorithm II model, the cumulative exit vehicle counts on $I_{11}, I_{12}, I_{15}, I_{16}$, which are most impacted by spillbacks. We can see that this continuum model approximates well its on-and-off counterpart.
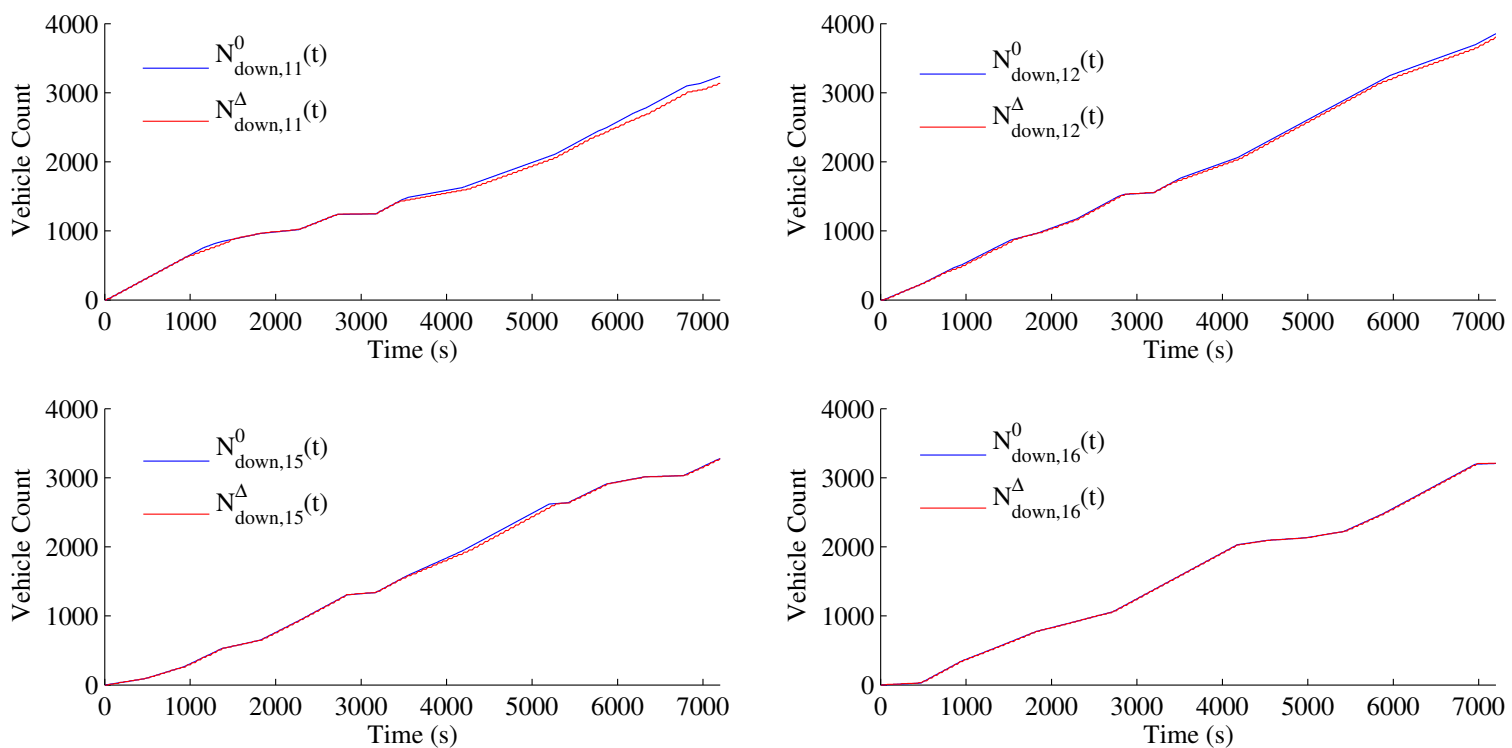

Figure 13: Comparison of the GCSM+Algorithm II model and the on-and-off model: Cumulative exit vehicle counts on four links.

Table 3 also suggests that the computational efficiency of these three continuum models is quite similar, and far more improved than the on-and-off model, which takes $0.43 \mathrm{~s}$ on average for the same simulation task. This is, again, due to the larger time steps allowed by the continuum models in order to implement a given signal timing plan.

\subsection{Signal optimization based on metaheuristics}

We present a signal optimization problem on the corridor shown in Figure 14, which is a subnetwork of the one in Figure 12. All link parameters remain the same. The signal cycles at the two nodes $F$ and $G$ are fixed to be 60 seconds. The decision variables of this optimization problem include the green splits and the offset of these two signals:

$$
\eta_{11} \in[0.2,0.8], \quad \eta_{12} \in[0.2,0.8], \quad O_{11,12} \in[0,60)
$$

where 0.2 and 0.8 are specified as the lower and upper bounds on the splits. By default, we assume that $\eta_{15}=1-\eta_{11}$, $\eta_{16}=1-\eta_{12}$. The objective function of this optimization problem is specified as 


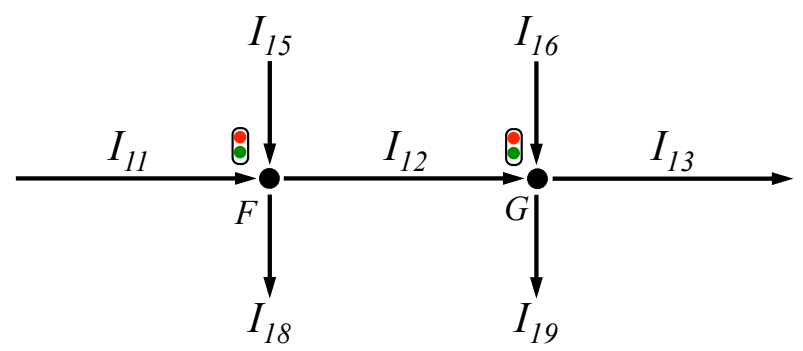

Figure 14: The network subject to signal optimization.

$$
\max \sum_{k=1}^{N}(N+1-k)\left(f_{\text {out }, 13}\left(t_{k}\right)+f_{\text {out }, 18}\left(t_{k}\right)+f_{\text {out }, 19}\left(t_{k}\right)\right) \cdot d t
$$

where $f_{\text {out }, i}\left(t_{k}\right)$ represents the discrete-time exit flow at time step $t_{k}, d t$ is the step size. Such an objective function maximizes the network throughput and minimize delay at each time step. Note that other types of objectives can be equally considered but are not the main focus of this numerical study.

The time horizon of the problem is one hour. The network inflows are generated randomly between zero and the flow capacity, and are given a priori as input; for example, they may be obtained from historical traffic flow data. Regarding the optimization method, We employ the particle swarm optimization (PSO) technique (Kennedy and Eberhart, 1995; Banks et al., 2007), noting that other types of metaheuristics may be applied as well with minimum modification. Due to space limitation, details of the optimization algorithm will be omitted here, and we refer the reader to the two references mentioned above. The PSO requires iteratively evaluating the objective value through simulation with given signal parameters.

The continuum signal model (GCSM+Algorithm II) and the on-and-off model are implemented on this network, with a time step size of $15 \mathrm{~s}$ and $5 \mathrm{~s}$ respectively. Note that $15 \mathrm{~s}$ (the link free-flow time) is the largest time step allowed by the link transmission model; and the time step for the on-and-off model should not be too large, otherwise the signal control resolution will be too low. Given these time steps the computational times for one simulation run are $0.02 \mathrm{~s}$ (continuum) and $0.09 \mathrm{~s}$ (on-and-off). The results of the PSO-based optimization are summarized in Table 5 , from which we observe that:

1. The continuum approach is far more efficient than the on-and-off approach, due to its flexibility in choosing larger time steps.

2. The decision resolution of the continuum model is higher (the green times and offset can be any real numbers) than the on-and-off model (the green times and offset can only be multiples of the step size 5s).

\begin{tabular}{|c|c|c|c|c|c|}
\hline & CPU time & Iteration \# & Green for $I_{11}$ & Green for $I_{12}$ & $O_{11,12}^{*}$ \\
\hline Continuum & $207 \mathrm{~s}$ & 23 & $26.4 \mathrm{~s}$ & $27.4 \mathrm{~s}$ & $34.0 \mathrm{~s}$ \\
\hline On-and-off & $1516 \mathrm{~s}$ & 31 & $25 \mathrm{~s}$ & $30 \mathrm{~s}$ & $35 \mathrm{~s}$ \\
\hline
\end{tabular}

Table 5: Results of the heuristic optimization.

To further verify that the higher decision resolution offered by the continuum model indeed brings improvement in the objective value, we use the on-and-off model to simulate the network using signal parameters from the table. A time step of $1 \mathrm{~s}$ is used (the green times provided by the continuum model are rounded to the nearest integer). The resulting objectives are $1.1122 \times 10^{7}$ (using parameters from the first row) and $1.0916 \times 10^{7}$ (using parameters from the second row), which indeed shows an improvement by $1.89 \%$. We are able to conclude that applying the continuum model not only brings substantial savings in computational time, but may also improve the solution quality by allowing a finer control resolution. 
ARTICLE LINK: http://www.sciencedirect.com/science/article/pii/S019126151500048X

PLEASE CITE THIS ARTICLE AS

Han, K., Gayah, V.V., 2015. Continuum signalized junction model for dynamic traffic networks: Offset, spillback, and multiple signal phases. Transportation Research Part B 77, 213-239.

\section{Summary and conclusion}

This paper presents significant extensions to the continuum signalized intersection model that was exhaustively studied in Han et al. (2014) to improve the representation of traffic operations under several realistic cases - specifically, the existence of offsets between adjacent signals, both sustained and transient queue spillbacks between closely spaced signals, and complex junction movements and phasing schemes. These extensions are derived theoretically, and bounds for the errors that would arise between the proposed model and the more realistic on-and-off signalized intersection model are provided using the LWR model of kinematic waves. The model extension for the treatment of signal offsets in the absence of spillback adjusts curves of cumulative vehicle counts at downstream intersections based on deviation of the offset from the free-flow travel time. For queue spillbacks, the generalized continuum signal model (GCSM) incorporates the blockage effects from downstream links using knowledge of the relative signal phases and speed of backward-moving waves along these links. The GCSM is supported by two methods to estimate the supply functions of links that trigger spillback. Overall, these extensions are shown to significantly improve upon the original model presented in Han et al. (2014). For one, the errors under queue spillbacks in the extended continuum signalized intersection model can now be bounded by fixed values that do not grow with time (e.g. GCSM+Algorithm II), unlike the original model. For another, the proposed models can explicitly handle complex spillback patterns that affect a sequence of junctions. In comparison, the original continuum model (Han et al., 2014) ignores any knowledge of signal offsets and thus could not be used to accurately assess operations on coordinated signalized arterials. We have also qualitatively described how the extended continuum model can be applied to more realistic intersection configurations that include multiple entry and exit links, complex signal phasing schemes, all-red times and dedicated turning lanes. These qualitative extensions allow the continuum model to be applied to most scenarios that are typically expected on urban traffic networks.

Numerical examples of the extensions confirm that the extended continuum model is able to approximate the behavior of the more realistic on-and-off signal model with remarkable consistency. In addition, this model is much more computationally efficient than the traditional on-and-off models in simulation tasks, and leads to higher signal control resolutions in a decision-making environment. This suggests that the continuum model is a promising approach to estimate traffic conditions on large-scale urban networks for a variety of purposes.

It is important to note, however, that the appropriateness of the continuum model for any modeling exercise will depend on the specific goals being considered. The small errors that arise with respect to the on-and-off model might be significant for some applications, particularly when detailed information is required about operations at a specific intersection. Instead, the continuum model is more appropriate for studying the aggregate behavior of vehicles on large-scale urban networks made up of many individual signalized intersections in close proximity. Users of the model should carefully consider the potential errors that might exist to decide if the precision of the model is appropriate for a given scenario.

One area for which the continuum signalized intersection model shows great promise is the integration with the network performance sub-model of dynamic traffic assignment (DTA) problems. These DTA models tend to be computationally intensive exercises that are hampered by the discontinuous nature of travel time functions caused by the on-and-off signal behavior. The continuum model, in contrast, provides smooth, continuous travel time functions that are obtained with much less computational effort. Thus, it is well-suited for integration within these larger DTA frameworks. The continuum model is also extremely useful in large-scale signal timing optimization efforts due to its superior computational efficiency over the on-and-off model. Although it represents traffic dynamics in an approximate way, the relatively small errors in aggregate traffic dynamics allows optimal solutions to be obtained relatively easily for large networks made up of many signals. Detailed optimization procedures can then be used to refine these initial solutions, as described qualitatively in Daganzo et al. (2012). Preliminary work on these applications are currently underway.

\section{Appendix A. Variational formulation in the absence of spillback}

In this section we derive the variational formulation under the assumption of no spillback. Let the fundamental diagram be triangular or trapezoidal. We claim that the resulting flow dynamic resembles that of a point-queue model, when no spillback is present. Indeed, according to the classical variational theory (Newell, 1993) for the triangular or 
ARTICLE LINK: http://www.sciencedirect.com/science/article/pii/S019126151500048X

PLEASE CITE THIS ARTICLE AS

Han, K., Gayah, V.V., 2015. Continuum signalized junction model for dynamic traffic networks: Offset, spillback, and multiple signal phases. Transportation Research Part B 77, 213-239.

trapezoidal fundamental diagram, we have that

$$
D(t)= \begin{cases}C & \text { if } N_{\text {up }}(t-\mathcal{T})>N_{\text {down }}(t) \\ f_{\text {in }}(t-\mathcal{T}) & \text { if } N_{\text {up }}(t-\mathcal{T})=N_{\text {down }}(t)\end{cases}
$$

where $D(\cdot)$ denotes the demand of the link; $N_{u p}(\cdot)$ and $N_{\text {down }}(\cdot)$ denote the cumulative entering and exiting vehicle counts, respectively; $f_{\text {in }}(\cdot)$ denotes the link inflow; $C$ and $\mathcal{T}$ denote, respectively, the flow capacity and the free-flow travel time. Here, $N_{u p}(t-\mathcal{T})-N_{\text {down }}(t)$ represents the queue volume on this link; for reason that will soon become clear, we use $q(t)$ to denote this quantity. Assuming infinite downstream receiving capacity, Eqn (A.1) leads to the following:

$$
f_{\text {out }}(t)=\left\{\begin{array}{ll}
C & \text { if } q(t)>0 \\
f_{\text {in }}(t-\mathcal{T}) & \text { if } q(t)=0
\end{array}, \quad q(t)=N_{\text {up }}(t-\mathcal{T})-N_{\text {down }}(t)\right.
$$

which is immediately recognized as the Vickrey model (Vickrey, 1969; Han et al., 2013a,b). In Han et al. (2013a), a formula for the generalized Vickrey model with possibly time-varying downstream receiving capacity, $M(t)$, is given as follows.

$$
N_{\text {down }}(t)=\min _{\tau \leq t}\left\{N_{u p}(\tau-\mathcal{T})-V(\tau)\right\}+V(t)
$$

where $V(t) \doteq \int_{0}^{t} M(\tau) d \tau . V(t)$ is interpreted as a weak downstream boundary condition (Aubin et al., 2008; Claudel and Bayen, 2010).

\section{Appendix B. Proof of theorems}

Appendix B.1. Proof of Theorem 3.2

Proof. Applying (3.6) to link $I_{2}$ yields the following calculation.

$$
\begin{aligned}
N_{\text {down }, 2}^{\Delta}(t) & =\min _{\tau \leq t}\left\{N_{u p, 2}^{\Delta}\left(\tau-\mathcal{T}_{2}\right)-V_{2}^{\Delta}(\tau)\right\}+V_{2}^{\Delta}(t)=\min _{\tau \leq t}\left\{N_{\text {down }, 1}^{\Delta}\left(\tau-\mathcal{T}_{2}\right)-V_{2}^{\Delta}(\tau)\right\}+V_{2}^{\Delta}(t) \\
& =\min _{\tau \leq t}\{\underbrace{\min _{\xi \leq \tau-\mathcal{T}_{2}}\left\{N_{u p, 1}^{\Delta}\left(\xi-\mathcal{T}_{1}\right)-V_{1}^{\Delta}(\xi)\right\}}_{F(\tau)}+\underbrace{V_{1}^{\Delta}\left(\tau-\mathcal{T}_{2}\right)-V_{2}^{\Delta}(\tau)}_{\delta(\tau)}\}+V_{2}^{\Delta}(t)
\end{aligned}
$$

where $F(\tau)$ is a monotonically decreasing function. Similarly, we can derive the following:

$$
\begin{aligned}
N_{\text {down }, 2}^{0}(t) & =\min _{\tau \leq t}\left\{\min _{\xi \leq \tau-\mathcal{T}_{2}}\left\{N_{u p, 1}^{0}\left(\xi-\mathcal{T}_{1}\right)-V_{1}^{0}(\xi)\right\}+V_{1}^{0}\left(\tau-\mathcal{T}_{2}\right)-V_{2}^{0}(\tau)\right\}+V_{2}^{0}(t) \\
& =\min _{\tau \leq t}\left\{\min _{\xi \leq \tau-\mathcal{T}_{2}}\left\{N_{u p, 1}^{0}\left(\xi-\mathcal{T}_{1}\right)-V_{1}^{0}(\xi)\right\}\right\}-\mathcal{T}_{2} \frac{\mathcal{M}}{\Delta}+V_{2}^{0}(t) \\
& =\min _{\xi \leq t-\mathcal{T}_{2}}\left\{N_{u p, 1}^{0}\left(\xi-\mathcal{T}_{1}\right)-V_{1}^{0}(\xi)\right\}+V_{1}^{0}\left(t-\mathcal{T}_{2}\right)=N_{\text {down }, 1}^{0}\left(t-\mathcal{T}_{2}\right)
\end{aligned}
$$

Here, we have used the fact that $V_{1}^{0}(t)=V_{2}^{0}(t)=\frac{\mathcal{M}}{\Delta} t$, where $\Delta$ is the common cycle time and $\mathcal{M}$ is the common maximum throughput per cycle. Next, we claim that

$$
0 \leq N_{d o w n, 1}^{\Delta}\left(t-\mathcal{T}_{2}\right)-N_{d o w n, 2}^{\Delta}(t) \leq \delta(t)
$$

Indeed, on one hand we have by (B.1) that

$$
\begin{aligned}
N_{\text {down }, 1}^{\Delta}\left(t-\mathcal{T}_{2}\right)-N_{\text {down }, 2}^{\Delta}(t) & =\min _{\tau \leq t-\mathcal{T}_{2}}\left\{N_{u p, 1}^{\Delta}\left(\tau-\mathcal{T}_{1}\right)-V_{1}^{\Delta}(\tau)\right\}+V_{1}^{\Delta}\left(t-\mathcal{T}_{2}\right)-N_{\text {down }, 2}^{\Delta}(t) \\
& =F(t)+V_{1}^{\Delta}\left(t-\mathcal{T}_{2}\right)-\min _{\tau \leq t}\{F(\tau)+\delta(\tau)\}-V_{2}^{\Delta}(t) \\
& =F(t)+\delta(t)-\min _{\tau \leq t}\{F(\tau)+\delta(\tau)\} \geq 0
\end{aligned}
$$


ARTICLE LINK: http://www.sciencedirect.com/science/article/pii/S019126151500048X

PLEASE CITE THIS ARTICLE AS

Han, K., Gayah, V.V., 2015. Continuum signalized junction model for dynamic traffic networks: Offset, spillback, and multiple signal phases. Transportation Research Part B 77, 213-239.

On the other hand, fix any $t$, we denote $\tau^{*} \doteq \underset{\tau \leq t}{\operatorname{argmin}}\{F(\tau)+\delta(\tau)\} \leq t$. Then

$$
F(t)+\delta(t)-\min _{\tau \leq t}\{F(\tau)+\delta(\tau)\}=F(t)-F\left(\tau^{*}\right)+\delta(t)-\delta\left(\tau^{*}\right) \leq \delta(t)
$$

due to the fact that $F(\cdot)$ is decreasing and the fact that $\delta(\cdot) \geq 0$. This establishes our claim. Finally, in view of (B.2), (B.3) and the following result from Han et al. (2014):

$$
\left|N_{\text {down }, 1}^{0}(t)-N_{\text {down,1 }}^{\Delta}(t)\right| \leq \eta_{1}\left(1-\eta_{1}\right) \Delta \mathcal{E}_{1} \quad \forall t
$$

we must have that

$$
\begin{aligned}
N_{\text {down }, 2}^{0}(t)-N_{\text {down }, 2}^{\Delta}(t) & =N_{\text {down }, 1}^{0}\left(t-\mathcal{T}_{2}\right)-N_{\text {down }, 2}^{\Delta}(t) \\
& \geq-\eta_{1}\left(1-\eta_{1}\right) \Delta \mathcal{E}_{1}+N_{\text {down }, 1}^{\Delta}\left(t-\mathcal{T}_{2}\right)-N_{\text {down }, 2}^{\Delta}(t) \geq-\eta_{1}\left(1-\eta_{1}\right) \Delta \mathcal{E}_{1}
\end{aligned}
$$

and that

$$
\begin{aligned}
N_{\text {down }, 2}^{0}(t)-N_{\text {down }, 2}^{\Delta}(t) & =N_{\text {down }, 1}^{0}\left(t-\mathcal{T}_{2}\right)-N_{\text {down }, 2}^{\Delta}(t) \\
& \leq \eta_{1}\left(1-\eta_{1}\right) \Delta \mathcal{E}_{1}+N_{\text {down }, 1}^{\Delta}\left(t-\mathcal{T}_{2}\right)-N_{\text {down }, 2}^{\Delta}(t) \leq \eta_{1}\left(1-\eta_{1}\right) \Delta \mathcal{E}_{1}+\delta(t)
\end{aligned}
$$

\section{Appendix B.2. Proof of Theorem 4.6}

Proof. The key observation is that with the same initial and upstream boundary conditions, the difference in the Moskowitz functions is bounded by the maximum difference in the weak downstream boundary conditions:

$$
V^{0}(t) \doteq \int_{0}^{t} R d \tau, \quad V^{\Delta}(t) \doteq \int_{0}^{t} u(\tau) \cdot \mathcal{E}(\tau) d \tau
$$

Both integrands above are periodic with period $\Omega$, and the following equality holds by definition

$$
\int_{t_{0}}^{t_{0}+\Omega} R d \tau=\int_{t_{0}}^{t_{0}+\Omega} u(\tau) \cdot \mathcal{E}(\tau) d \tau
$$

where $t_{0}$ is arbitrary. Thus it suffices for us to inspect the maximum difference between $V^{0}(t)$ and $V^{\Delta}(t)$ for $t \in$ $\left[t_{0}, t_{0}+\Omega\right]$. Define $j^{*} \doteq \max \left\{0 \leq j \leq m: K_{j} \leq R\right\}$. Given that both $V^{0}(\cdot)$ and $V^{\Delta}(\cdot)$ are piecewise affine, we have

$$
\max _{t \in\left[t_{0}, t_{0}+\Omega\right]}\left|V^{0}(t)-V^{\Delta}(t)\right| \leq R \sum_{j=0}^{j^{*}} \mu\left(\beta_{j}\right)-\sum_{j=0}^{j^{*}} \mu\left(\beta_{j}\right) \cdot K_{j},
$$

which is (4.22).

\section{Appendix B.3. Proof of Theorem 4.12}

Proof. In view of Figure 6, applying Method I to $I_{10}$ with inputs $u_{10}(t), \mathcal{E}_{10}(t) \equiv C_{10}$ and $\Omega_{10}=\Delta_{10}$ yields the following estimate:

$$
\begin{aligned}
& \left|\int_{t_{0}}^{t_{0}+L C M\left(\Delta_{8}, \Delta_{10}\right)} u_{8}(\tau)\left(\mathcal{E}_{8}(\tau)-\tilde{\mathcal{E}}_{8}(\tau)\right) d \tau\right| \leq \int_{t_{0}}^{t_{0}+L C M\left(\Delta_{8}, \Delta_{10}\right)}\left|\mathcal{E}_{8}(\tau)-\tilde{\mathcal{E}}_{8}(\tau)\right| d \tau \\
\leq & \frac{1}{2}\left(1-\eta_{10}\right) \Delta_{10} \min \left\{C_{8}, C_{10}\right\} \cdot \frac{L C M\left(\Delta_{8}, \Delta_{10}\right)}{\Delta_{10}}=\frac{1}{2}\left(1-\eta_{10}\right) \min \left\{C_{8}, C_{10}\right\} L C M\left(\Delta_{8}, \Delta_{10}\right) \doteq A
\end{aligned}
$$


ARTICLE LINK: http://www.sciencedirect.com/science/article/pii/S019126151500048X

PLEASE CITE THIS ARTICLE AS

Han, K., Gayah, V.V., 2015. Continuum signalized junction model for dynamic traffic networks: Offset, spillback, and multiple signal phases. Transportation Research Part B 77, 213-239.

where $t_{0}$ is the beginning of an arbitrary cycle of $u_{10}(\cdot) \cdot \mathcal{E}_{10}(\cdot) . \mathcal{E}_{8}(t)=\min \left\{C_{8}, S_{10}(t)\right\}, \tilde{\mathcal{E}}_{8}(t)=\min \left\{C_{8}, \tilde{S}_{10}(t)\right\}$ where $S_{10}(t)$ is the exact supply function and $\tilde{S}_{10}(t)$ is the supply function estimated by Algorithm I. On the other hand, we have

$$
\begin{aligned}
\left|\int_{t_{0}}^{t_{0}+L C M\left(\Delta_{8}, \Delta_{10}\right)} u_{8}(\tau)\left(\mathcal{E}_{8}(\tau)-\tilde{\mathcal{E}}_{8}(\tau)\right) d \tau\right| & \leq \min \left\{C_{8}, C_{10}\right\}\left|\int_{t_{0}}^{t_{0}+L C M\left(\Delta_{8}, \Delta_{10}\right)} u_{8}(\tau) d \tau\right| \\
& =\min \left\{C_{8}, C_{10}\right\} L C M\left(\Delta_{8}, \Delta_{10}\right) \eta_{8} \doteq B
\end{aligned}
$$

Combining the two estimates above, we have

$$
\left|\int_{t_{0}}^{t_{0}+L C M\left(\Delta_{8}, \Delta_{10}\right)} u_{8}(\tau)\left(\mathcal{E}_{8}(\tau)-\tilde{\mathcal{E}}_{8}(\tau)\right) d \tau\right| \leq \min \{A, B\}
$$

Define the weak downstream boundary conditions

$$
V_{8}^{\Delta}(t) \doteq \int_{0}^{t} u_{8}(\tau) \cdot \mathcal{E}_{8}(\tau) d \tau, \quad \tilde{V}_{8}^{\Delta}(t) \doteq \int_{0}^{t} u_{8}(\tau) \cdot \tilde{\mathcal{E}}_{8}(\tau) d \tau
$$

It follows from (B.6) that $\forall t \gg \operatorname{LCM}\left(\Delta_{8}, \Delta_{10}\right)$,

$$
\begin{aligned}
\left|V_{8}^{\Delta}(t)-\tilde{V}_{8}^{\Delta}(t)\right| & =\left|\int_{0}^{t} u_{8}(\tau) \cdot\left(\mathcal{E}_{8}(\tau)-\tilde{\mathcal{E}}_{8}(\tau)\right) d \tau\right| \leq\left\lceil\frac{t}{L C M\left(\Delta_{8}, \Delta_{10}\right)}\right\rceil \cdot \min \{A, B\} \\
& \approx \min \left\{\frac{1}{2}\left(1-\eta_{10}\right) \min \left\{C_{8}, C_{10}\right\}, \min \left\{C_{8}, C_{10}\right\} \eta_{8}\right\} t
\end{aligned}
$$

where $\lceil x\rceil$ is the smallest integer that is greater than or equal to $x \in \mathbb{R}$. According to (4.20), the weak downstream boundary condition corresponding to the GCSM is

$$
V_{8}^{0}(t) \doteq \int_{0}^{t} \zeta_{8} \min \left\{C_{8}, C_{10}\right\} d \tau=\zeta_{8} \min \left\{C_{8}, C_{10}\right\} \cdot t
$$

where $\zeta_{8}$ is the green split of the $\operatorname{ESC} \mathbf{u}_{8}(t)=u_{8}(t) \cdot \tilde{\mathcal{E}}_{8}(t) / C_{8}$. As a special case of (4.22), we have that

$$
\left|V_{8}^{0}(t)-\tilde{V}_{8}^{\Delta}(t)\right| \leq \zeta_{8}\left(1-\zeta_{8}\right) L C M\left(\Delta_{8}, \Delta_{10}\right) \min \left\{C_{8}, C_{10}\right\}
$$

Finally, given the same initial or upstream boundary conditions, the difference in the Moskowitz functions is bounded by the maximum difference in the weak downstream boundary conditions. And we have

$$
\left|N_{8}^{0}(t, x)-N_{8}^{\Delta}(t, x)\right| \leq \max _{t}\left|V_{8}^{0}(t)-V_{8}^{\Delta}(t)\right| \leq \max _{t}\left|V_{8}^{0}(t)-\tilde{V}_{8}^{\Delta}(t)\right|+\max _{t}\left|V_{8}^{\Delta}(t)-\tilde{V}_{8}^{\Delta}(t)\right|
$$

Combining this with (B.8) and (B.9) yields the desired result.

\section{Appendix C. Derivation of the algebraic equation system in Algorithm II}

Under the same setting and notation as in Algorithm II, we observe from Figure C.15 that the value of $S(t)$ for $t \in\left[t_{0}+\frac{L}{w}, t_{0}+\frac{L}{w}+\operatorname{LCM}\left(\Omega, \Delta_{u p}\right)\right]$ is influenced only by the inflow $f_{i n}^{\Delta}(t), t \in\left[t_{0}+\frac{L}{w}, t_{0}+\frac{L}{w}+L C M\left(\Omega, \Delta_{u p}\right)\right]$ and the outflow $f_{\text {out }}^{\Delta}(t), t \in\left[t_{0}, t_{0}+\operatorname{LCM}\left(\Omega, \Delta_{u p}\right)\right]$. With this in mind, we impose the following virtual initial condition that is consistent with the configuration in Figure C.15:

$$
\rho\left(t_{0}, x\right) \equiv \rho^{j a m} \quad \forall x \in[a, b]
$$

without affecting $S(t)$ for $t \in\left[t_{0}+\frac{L}{w}, t_{0}+\frac{L}{w}+L C M\left(\Omega, \Delta_{u p}\right)\right]$. We then apply the variational principle (Newell, 1993; Han et al., 2014) to get, for all $t \in\left[t_{0}+\frac{L}{w}, t_{0}+\frac{L}{w}+\operatorname{LCM}\left(\Omega, \Delta_{u p}\right)\right]$,

$$
S(t)= \begin{cases}f_{\text {out }}^{\Delta}\left(t-\frac{L}{w}\right) & \text { if } \rho^{j a m} L+\int_{t_{0}+\frac{L}{w}}^{t} f_{\text {in }}^{\Delta}(\tau) d \tau=\int_{t_{0}}^{t-\frac{L}{w}} f_{\text {out }}^{\Delta}(\tau) d \tau+\rho^{j a m} L \\ C & \text { if } \rho^{j a m} L+\int_{t_{0}+\frac{L}{w}}^{t} f_{\text {in }}^{\Delta}(\tau) d \tau<\int_{t_{0}}^{t-\frac{L}{w}} f_{\text {out }}^{\Delta}(\tau) d \tau+\rho^{j a m} L\end{cases}
$$




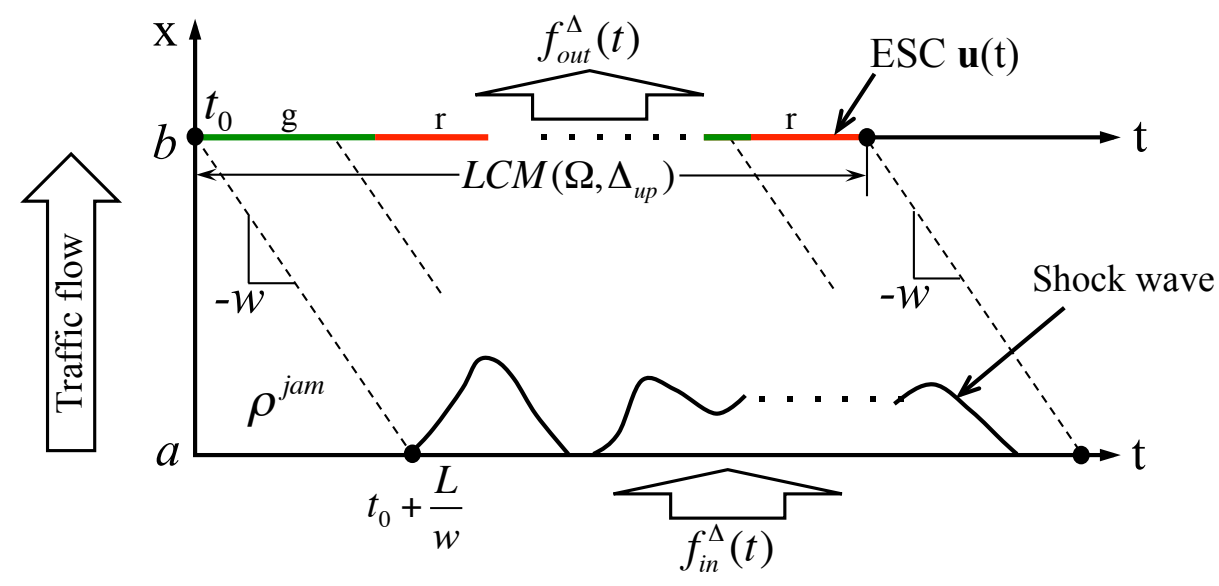

Figure C.15: Calculation of the general supply function using the variational principle.

Further notice that since the exit of this link remains congested, $D(t) \equiv C$. Thus

$$
f_{\text {out }}^{\Delta}(t)=\min \{D(t), u(t) \cdot \mathcal{E}(t)\} \equiv u(t) \cdot \mathcal{E}(t)
$$

Substituting $f_{\text {out }}^{\Delta}(\cdot)$ in Eqn $($ C.1) with $u(\cdot) \cdot \mathcal{E}(\cdot)$ yields the algebraic equation system in Algorithm II.

\section{References}

Aboudolas, K., Papageorgiou, M. and Komsmatopoulous, E., 2009. Store-and-forward based methods for the signal control problem in large-scale congested urban road networks. Transportation Research Part C, 17 (2), 163-174.

Aubin, J.P., Bayen, A.M., Saint-Pierre, P., 2008. Dirichlet problems for some Hamilton-Jacobi equations with inequality constraints. SIAM Journal on Control and Optimization 47 (5), 2348-2380.

Aziz, H.M., Ukkusuri, S.V., 2012. Unified framework for dynamic traffic assignment and signal control with cell transmission model. Transportation Research Record, 2311, 73-84.

Banks, A., J. Vincent, C. Anyakoha, 2007. A review of particle swarm optimization. Part I: background and development. Natural Computing 6 (4), 467-484.

Cassidy, M.J., 1998. Bivariate relations in nearly stationary highway traffic. Transportation Research Part B 32(1), 49-59.

Ceylan, H., Bell, M.G.H., 2004. Traffic signal timing optimisation based on genetic algorithm approach, including driverss' routing. Transportation Research Part B, 38 (4), 329-342.

Chitour, Y., Piccoli, B., 2005. Traffic circles and timing of traffic lights for cars flow. Discrete and Continuous Dynamical Systems - Series B 5 (3), 599-630.

Chiu, S., Chand, S., 1993. Adaptive traffic signal control using fuzzy logic. Proceedings of the Second IEEE International Conference on Fuzzy Systems, 1371-1376.

Claudel, C.G., Bayen, A.M., 2010. Lax-Hopf Based incorporation of internal boundary conditions into Hamilton-Jacobi equation. Part I: Theory. IEEE Transactions on Automatic Control 55 (5), 1142-1157.

Daganzo, C.F., 1994. The cell transmission model: A simple dynamic representation of highway traffic. Transportation Research Part B 28 (4), 269-287.

Daganzo, C.F., 1995. The cell transmission model, part II: network traffic. Transportation Research Part B 29 (2), $79-93$.

Daganzo, C., Gayah, V., Gonzales, E., 2012. The potential of parsimonious models for understanding large scale transportation systems and answering big picture questions. EURO Journal of Transportation and Logistics 1 (1-2), 47-65.

Foy, M.D., Benekohal, R.F., Goldberg, D.E., 1992. Signal timing determination using genetic algorithms. Transportation Research Record 1365, 108-115.

Friesz, T.L., Bernstein, D., Smith, T., Tobin, R., Wie, B., 1993. A variational inequality formulation of the dynamic network user equilibrium problem. Operations Research 41 (1), 80-91.

Friesz, T.L., Han, K., Neto, P.A., Meimand, A., Yao, T., 2013. Dynamic user equilibrium based on a hydrodynamic model. Transportation Research Part B 47, 102-126.

Gazis, D.C and Potts, R.B., 1963. The oversatured intersection. In the proceedings of the Second International Symposium on Traffic Theory, London, UK.

Gayah, V.V., Daganzo, C.F., 2012. Analytical capacity comparison of one-way and two-way signalized street networks. Transportation Research Record 2301, 76-85.

Ge, Y.E., Zhou, X., 2012. An alternative definition of dynamic user optimum on signalised road networks. Journal of Advanced Transportation 46 (3), 236-253. 


\section{ARTICLE LINK: http://www.sciencedirect.com/science/article/pii/S019126151500048X}

\section{PLEASE CITE THIS ARTICLE AS}

Han, K., Gayah, V.V., 2015. Continuum signalized junction model for dynamic traffic networks: Offset, spillback, and multiple signal phases. Transportation Research Part B 77, 213-239.

Gu, W., Gayah, V.V., Cassidy, M.J., Saade, N., 2014. On the impacts of bus stops near signalized intersections: Models of car and bus delays. Transportation Research Part B 68, 123-140.

Guler, S.I., Cassidy, M.J., 2012. Strategies for sharing bottleneck capacity among buses and cars. Transportation Research Part B 46 (10), 13341345.

Han, K., 2013. An analytical approach to sustainable transportation network design. Ph.D. dissertation, Pennsylvania State University.

Han, K., Friesz, T.L., Yao, T., 2013a. A partial differential equation formulation of Vickrey's bottleneck model, part I: Methodology and theoretical analysis. Transportation Research Part B 49, 55-74.

Han, K., Friesz, T.L., Yao, T., 2013b. A partial differential equation formulation of Vickrey's bottleneck model, part II: Numerical analysis and computation. Transportation Research Part B 49, 75-93.

Han, K., Gayah, V., Piccoli, B., Friesz, T.L., Yao, T., 2014. On the continuum approximation of the on-and-off signal control on dynamic traffic networks. Transportation Research Part B 61, 73-97.

Improta, G., Cantarella, G.E., 1984. Control system design for an individual signalized junction. Transportation Research Part B 18 (2), $147-167$.

Kennedy, J., Eberhart, R., 1995. Particle swarm optimization. Proceedings of IEEE International Conference on Neural Networks (Perth, Australia), IEEE Service Center, Piscataway, NJ.

Lebacque, J., Khoshyaran, M., 1999. Modeling vehicular traffic flow on networks using macroscopic models, in Finite Volumes for Complex Applications II, 551-558, Hermes Science Publishing, Paris, 1999.

Lighthill, M., Whitham, G., 1955. On kinematic waves. II. A theory of traffic flow on long crowded roads. Proceedings of the Royal Society of London: Series A 229, 317-345.

Lo, H., 1999a. A novel traffic signal control formulation. Transportation Research Part A 33 (6), 433-448.

Lo, H., 1999b. A cell-based traffic control formulation: strategies and benefits of dynamic timing plans. Transportation Science 35 (2), $148-164$.

Miller, A.J., 1963. Computer control system for traffic networks. Proceedings of the 2nd International Symposium on Theory of Traffic Flow, London, UK.

Moskowitz, K., 1965. Discussion of 'freeway level of service as influenced by volume and capacity characteristics' by D.R. Drew and C.J. Keese. Highway Research Record 99, 43-44.

Murat, Y.S., Gedizlioglu, E., 2005. A fuzzy logic multi-phased signal control model for isolated junctions. Transportation Research Part C 13 (1),19-36.

Newell, G. F. (1993). A simplified theory of kinematic waves in highway traffic, parts I-III. Transportation Research Part B 27(4), $281-313$.

Richards, P.I., 1956. Shockwaves on the highway. Operations Research 4 (1), 42-51.

Robertson, D.J., Bretherton, R.D., 1974. Optimal control of an intersection for any known sequences of vehicle arrivals. Proceedings of the 2nd IFAC-IFIP-IFORS Sumposium on Traffic Control and Transport Systems, Monte Carlo.

Shelby, S., 2004. Single-intersection evaluation of real-time adaptive traffic signal control algorithms. Transportation Research Record 1867, 183192.

Smith M.J., 2010. Intelligent network control: using an assignment-control model to design fixed time signal timings. In New Developments in Transport Planning: Advances in Dynamic Traffic Assignment, Tempere CMJ, Viti F, Immers LH (eds). Edward Elgar: Cheltenham, UK, 57-71.

Szeto, W.Y., Lo, H.K., 2006. Dynamic traffic assignment: Properties and extensions. Transportmetrica 2 (1), 31-52.

TRB, 2000. Highway Capacity Manual. Transportation Research Board of the National Academies, Washington, DC.

Vickrey, W.S., 1969. Congestion theory and transport investment. The American Economic Review 59 (2), $251-261$.

Ukkusuri, S., Doan, K., Azzi, H.M, 2013. A bi-level formulation for the combined dynamic equilibrium based traffic signal control. Procedia Social and Behavioral Sciences 80, 729-752.

Yperman, I., S. Logghe and L. Immers, 2005. The Link Transmission Model: An Efficient Implementation of the Kinematic Wave Theory in Traffic Networks, Advanced OR and AI Methods in Transportation, Proc. 10th EWGT Meeting and 16th Mini-EURO Conference, Poznan, Poland, 122-127, Publishing House of Poznan University of Technology. 